\title{
REALISTIC COMPUTABLE ERROR BOUNDS FOR THREE DIMENSIONAL FINITE ELEMENT ANALYSES IN LINEAR ELASTICITY*
}

\author{
MARK AINSWORTH ${ }^{\dagger}$ AND RICHARD RANKIN $\ddagger$
}

\begin{abstract}
We obtain a computable estimator for the energy norm of the error in piecewise quadratic finite element approximations of linear elasticity in three dimensions. We show that the estimator provides guaranteed upper bounds on the energy norm of the error as well as (up to a constant and data oscillation terms) local lower bounds.
\end{abstract}

1. Introduction. Error estimators for linear elasticity problems go back at least as far as [16] with many more estimators being subsequently obtained for two dimensional linear elasticity $[7,8,11,12,18,19,24]$ and three dimensional linear elasticity $[10-13,17,20,24]$. However, the majority of estimators obtained are not actually computable since they involve either (a) generic unknown constants $[7,10-$ $12,24]$, or (b) the solution of (local) infinite dimensional problems (which cannot be solved exactly) $[8,13,19,20]$. Upper bounds can be obtained at the expense of the solution of a global finite element problem [17]. However, the only locally computable guaranteed error estimators for two dimensional linear elasticity problems, of which we are aware, are those in $[4,18]$.

A vital part of the analysis in both $[4,18]$ is the construction of a suitable field $\sigma_{K}$ whose normal components and divergence have certain properties. In [18] this field was constructed using the Arnold and Winther finite element [6] whilst in [4] the Arnold, Douglas and Gupta finite element [5] was used. In the current work we extend this result to three dimensional domains in the context of approximation on meshes comprised of tetrahedra. The computation of the estimator involves computations over patches of elements sharing a vertex, with the computation of the three dimensional version of the field $\boldsymbol{\sigma}_{K}$ constructed using a three dimensional analog of the Arnold, Douglas and Gupta element. We show that, if one wishes to avoid additional post-processing steps as in [18], then one is obliged to make use of so-called "macro-element" techniques and we believe the one presented here is the simplest one available.

Our estimator takes account of so-called "data oscillation" error at the expense of requiring an upper bound on the Korn's inequality on each element or on the domain itself. Suitable bounds in the two dimensional case were given in [14]. However, as far as we are aware, there is no three dimensional analog of the two dimensional result given in [14]. Of course, if the load data is piecewise affine (which is often the case in many stress analyses performed using finite elements), then there is no need for a bound on the constant in Korn's inequality, and our estimator provides a guaranteed upper bound on the energy norm of the error. We illustrate the performance of the estimator for a simple three dimensional test problem and show that the upper bound is both realistic, and suitable for driving an adaptive solution algorithm.

\section{Preliminaries.}

2.1. Model problem. Let $\lambda \geq 0$ and $\mu>0$ be given. Let $\Omega$ be a polyhedral domain whose boundary $\Gamma$ is partitioned into disjoint sets $\Gamma_{D}$ and $\Gamma_{N}$ on which the

\footnotetext{
* Support of the authors by the Engineering and Physical Sciences Research Council of Great Britain under the Numerical Algorithms and Intelligent Software (NAIS) for the evolving HPC platform grant EP/G036136/1 is gratefully acknowledged.

${ }^{\dagger}$ Department of Mathematics and Statistics, University of Strathclyde, 26 Richmond Street, Glasgow G1 1XH, Scotland. M.Ainsworth@strath.ac.uk.

${ }^{\ddagger}$ Department of Mathematics and Statistics, University of Strathclyde, 26 Richmond Street, Glasgow G1 1XH, Scotland. richard.a.rankin@strath.ac.uk.
} 
displacement and tractions are prescribed respectively. Let the spaces

$$
L_{2}(\Omega)=\left\{v:\|v\|_{L_{2}(\Omega)}^{2}=\int_{\Omega} v^{2} d \boldsymbol{x}<\infty\right\}
$$

and

$$
H^{1}(\Omega)=\left\{v: v \in L_{2}(\Omega), \operatorname{grad} v \in L_{2}(\Omega)^{2}\right\}
$$

For $\boldsymbol{v} \in \boldsymbol{H}^{1}(\Omega)=H^{1}(\Omega)^{3}$, let

$$
\underset{\approx}{\boldsymbol{\epsilon}}(\boldsymbol{v})=\frac{1}{2}\left(\underset{\approx}{\operatorname{grad}} \boldsymbol{v}+(\underset{\approx}{\operatorname{grad} v})^{T}\right)
$$

and

$$
\underset{\approx}{\boldsymbol{\sigma}}(\boldsymbol{v})=\underset{\approx}{\boldsymbol{\epsilon}}(\boldsymbol{v})=2 \mu \underset{\approx}{\boldsymbol{\epsilon}}(\boldsymbol{v})+\lambda \operatorname{tr}(\underset{\approx}{\boldsymbol{\epsilon}}(\boldsymbol{v})) \underset{\approx}{\boldsymbol{I}}
$$

denote the linearised strain and stress associated with a displacement $\boldsymbol{v}$, where $\underset{\widetilde{\sim}}{\boldsymbol{I}}$ is the identity tensor. The compliance tensor is given in terms of the inverse $\mathbb{E}^{-\widetilde{1}}$ defined by

$$
\mathbb{E}^{-1} \underset{\approx}{\boldsymbol{w}}=\frac{1}{2 \mu}\left(\underset{\approx}{\boldsymbol{w}}-\frac{\lambda}{2 \mu+3 \lambda} \operatorname{tr}(\underset{\approx}{\boldsymbol{w}}) \underset{\approx}{\boldsymbol{I}}\right)
$$

Consider the model problem of finding the linearly elastic displacement $\boldsymbol{u}$ such that

$$
\begin{aligned}
-\operatorname{div} \underset{\approx}{\boldsymbol{g}}(\boldsymbol{u}) & =\boldsymbol{f} \text { in } \Omega, \\
\boldsymbol{u} & =\boldsymbol{q} \text { on } \Gamma_{D}, \\
\underset{\approx}{\boldsymbol{g}}(\boldsymbol{u}) \boldsymbol{n}_{\Gamma} & =\boldsymbol{g} \text { on } \Gamma_{N},
\end{aligned}
$$

where $\boldsymbol{n}_{\Gamma}$ is the outward unit normal vector to $\Gamma$.

The variational form of this problem is to find $\boldsymbol{u} \in \boldsymbol{H}^{1}(\Omega)$ such that $\boldsymbol{u}=\boldsymbol{q}$ on $\Gamma_{D}$ and

$$
\int_{\Omega} \underset{\approx}{\boldsymbol{\sigma}}(\boldsymbol{u}): \underset{\approx}{\boldsymbol{\epsilon}}(\boldsymbol{v}) d \boldsymbol{x}=\int_{\Omega} \boldsymbol{f} \cdot \boldsymbol{v} d \boldsymbol{x}+\int_{\Gamma_{N}} \boldsymbol{g} \cdot \boldsymbol{v} d \boldsymbol{S} \forall \boldsymbol{v} \in \boldsymbol{H}_{D}^{1}(\Omega)
$$

where $\underset{\approx}{\boldsymbol{w}}: \underset{\approx}{\boldsymbol{v}}=\sum_{i=1}^{3} \sum_{j=1}^{3} w_{i j} v_{i j}$ and $\boldsymbol{H}_{D}^{1}(\Omega)=\left\{\boldsymbol{v} \in \boldsymbol{H}^{1}(\Omega): \boldsymbol{v}=\mathbf{0}\right.$ on $\left.\Gamma_{D}\right\}$.

The data are supposed to satisfy $\boldsymbol{f} \in \boldsymbol{L}_{2}(\Omega)=L_{2}(\Omega)^{3}$ and $\boldsymbol{g} \in \boldsymbol{L}_{2}\left(\Gamma_{N}\right)=$ $L_{2}\left(\Gamma_{N}\right)^{3}$. Likewise, we assume that the data $\boldsymbol{q}$ is smooth, and, in addition, is compatible in the sense that problem (2.1) admits a solution. We shall also assume that the boundary $\Gamma_{D}$ is such that a unique solution $\boldsymbol{u} \in \boldsymbol{H}^{1}(\Omega)$ to problem $(2.1)$ exists. In this case Korn's inequality

$$
\left(\int_{\Omega} \underset{\approx}{\operatorname{grad}} \boldsymbol{v}: \underset{\approx}{\operatorname{grad}} \boldsymbol{v} d \boldsymbol{x}\right)^{1 / 2} \leq C_{\Omega}\left(\int_{\Omega} \underset{\approx}{\boldsymbol{\epsilon}}(\boldsymbol{v}): \underset{\approx}{\boldsymbol{\epsilon}}(\boldsymbol{v}) d \boldsymbol{x}\right)^{1 / 2}
$$

is satisfied for all $\boldsymbol{v} \in \boldsymbol{H}_{D}^{1}(\Omega)$. For certain domains $\Omega$ and choices of $\Gamma_{D}$ an upper bound for the constant $C_{\Omega}$ is given in $[15,22]$. However, in many cases suitable bounds are unavailable and one can then resort to an approximation to $C_{\Omega}$ obtained by solving an eigenvalue problem. 
2.2. Partitioning the domain. Consider a family of partitions $\{\mathcal{P}\}$ of the domain $\Omega$ into the union of nonoverlapping, shape-regular tetrahedral elements such that the nonempty intersection of a distinct pair of elements is a single common node, single common edge or single common face which is an entire face of both of these elements. Consequently, the family of partitions is locally quasi-uniform in the sense that the ratio of the diameters of any pair of neighbouring elements is uniformly bounded above and below over the whole family. In addition, we shall insist that each element face $\gamma$ which lies on the domain boundary satisfies one of the inclusions $\gamma \subset \Gamma_{D}$ or $\gamma \subset \Gamma_{N}$.

Henceforth, we shall consider a fixed partition $\mathcal{P}$ from the family. Let $K$ and $K^{\prime}$ denote individual elements in $\mathcal{P}$, let $\partial K$ denote the boundary of element $K$ and let $\mathcal{F}_{K}$ denote the set containing the individual faces of element $K$. Likewise, we let $\mathcal{F}_{I}, \mathcal{F}_{D}$ and $\mathcal{F}_{N}$ denote the disjoint sets of faces defined by

$$
\begin{aligned}
\mathcal{F}_{I} & =\left\{\gamma: \gamma=\partial K \cap \partial K^{\prime}, K, K^{\prime} \in \mathcal{P}\right\}, \\
\mathcal{F}_{D} & =\left\{\gamma \subset \Gamma_{D}: \gamma \in \mathcal{F}_{K} \text { for some } K \in \mathcal{P}\right\}, \\
\mathcal{F}_{N} & =\left\{\gamma \subset \Gamma_{N}: \gamma \in \mathcal{F}_{K} \text { for some } K \in \mathcal{P}\right\}
\end{aligned}
$$

and let $\partial \mathcal{P}=\mathcal{F}_{I} \cup \mathcal{F}_{D} \cup \mathcal{F}_{N}$ denote the set of all element faces. For $m \in \mathbb{N}_{0}$, let $\mathbb{P}_{m}(K)$ denote the space of polynomials on $K \in \mathcal{P}$ of total degree at most $m$ and let $\mathbb{P}_{m}(\gamma)$ denote the space of polynomials on $\gamma \in \partial \mathcal{P}$ of total degree at most $m$. We also let $|K|$ denote the volume of the element $K$ and let $|\gamma|$ denote the area of face $\gamma$.

2.3. Finite element approximation. The conforming finite element space of second order $\boldsymbol{X} \subset \boldsymbol{H}^{1}(\Omega)$ is defined by

$$
\boldsymbol{X}=\left\{\boldsymbol{v} \in C(\bar{\Omega})^{3}: \boldsymbol{v}_{\mid K} \in \mathbb{P}_{2}(K)^{3} \forall K \in \mathcal{P}\right\},
$$

with the subspace $\boldsymbol{X}_{D} \subset \boldsymbol{H}_{D}^{1}(\Omega)$ being defined by

$$
\boldsymbol{X}_{D}=\left\{\boldsymbol{v} \in \boldsymbol{X}: \boldsymbol{v}=\mathbf{0} \text { on } \Gamma_{D}\right\} .
$$

The conforming finite element approximation of second order of problem (2.1) consists of finding $\boldsymbol{u}_{\boldsymbol{X}} \in \boldsymbol{X}$ such that

$$
\int_{\Omega} \underset{\sim}{\boldsymbol{g}}\left(\boldsymbol{u}_{\boldsymbol{X}}\right): \underset{\sim}{\boldsymbol{\epsilon}}(\boldsymbol{v}) d \boldsymbol{x}=\int_{\Omega} \boldsymbol{f} \cdot \boldsymbol{v} d \boldsymbol{x}+\int_{\Gamma_{N}} \boldsymbol{g} \cdot \boldsymbol{v} d \boldsymbol{S} \forall \boldsymbol{v} \in \boldsymbol{X}_{D}
$$

subject to the boundary condition $\boldsymbol{u}_{\boldsymbol{X}}=\boldsymbol{q}$ on $\Gamma_{D}$. For simplicity, we suppose that the Dirichlet conditions can be satisfied exactly using the finite element space.

2.4. Projections and oscillation of the data. For $K \in \mathcal{P}$, let $P_{K} \boldsymbol{f} \in$ $\mathbb{P}_{1}(K)^{3}$ be the function such that

$$
\int_{K}\left(\boldsymbol{f}-P_{K} \boldsymbol{f}\right) \cdot \boldsymbol{p} d \boldsymbol{x}=0 \text { for all } \boldsymbol{p} \in \mathbb{P}_{1}(K)^{3} .
$$

For $\gamma \in \mathcal{F}_{N}$, let $P_{\gamma} \boldsymbol{g} \in \mathbb{P}_{1}(\gamma)^{3}$ be the function such that

$$
\int_{\gamma}\left(\boldsymbol{g}-P_{\gamma} \boldsymbol{g}\right) \cdot \boldsymbol{p} d \boldsymbol{S}=0 \text { for all } \boldsymbol{p} \in \mathbb{P}_{1}(\gamma)^{3} .
$$

We define the oscillation of the data $\boldsymbol{f}$ on an element $K \in \mathcal{P}$ to be

$$
\operatorname{osc}(\boldsymbol{f}, K)=h_{K}\left\|\boldsymbol{f}-P_{K} \boldsymbol{f}\right\|_{\boldsymbol{L}_{2}(K)}
$$


where $h_{K}$ is the length of the longest edge of element $K$. The oscillation of the Neumann data $\boldsymbol{g}$ on a face $\gamma \in \mathcal{F}_{N}$ is defined to be

$$
\operatorname{osc}(\boldsymbol{g}, \gamma)=|\gamma|^{1 / 4}\left\|\boldsymbol{g}-P_{\gamma} \boldsymbol{g}\right\|_{\boldsymbol{L}_{2}(\gamma)} .
$$

3. Estimation of the energy norm of the error. Let the energy norm over a region $\omega$ be denoted by

$$
\|\cdot\|_{\omega}=\left(\int_{\omega} \underset{\approx}{\boldsymbol{\sigma}}(\cdot): \underset{\approx}{\boldsymbol{\epsilon}}(\cdot) d \boldsymbol{x}\right)^{1 / 2}
$$

We shall omit the subscript in the case where $\omega=\Omega$. We are interested in obtaining computable bounds for the error $\boldsymbol{e}=\boldsymbol{u}-\boldsymbol{u}_{X}$ measured in the energy norm.

Let $\boldsymbol{v} \in \boldsymbol{H}_{D}^{1}(\Omega)$. Then, by the definition of $\boldsymbol{e}$ and (2.1), there holds

$$
\begin{aligned}
\int_{\Omega} \underset{\approx}{\boldsymbol{\sigma}}(\boldsymbol{e}): \underset{\approx}{\boldsymbol{\epsilon}}(\boldsymbol{v}) d \boldsymbol{x} & =\int_{\Omega} \underset{\approx}{\boldsymbol{\sigma}}(\boldsymbol{u}): \underset{\approx}{\boldsymbol{\epsilon}}(\boldsymbol{v}) d \boldsymbol{x}-\int_{\Omega} \underset{\approx}{\boldsymbol{\sigma}}\left(\boldsymbol{u}_{X}\right): \underset{\approx}{\boldsymbol{\epsilon}}(\boldsymbol{v}) d \boldsymbol{x} \\
& =\int_{\Omega} \boldsymbol{f} \cdot \boldsymbol{v} d \boldsymbol{x}+\int_{\Gamma_{N}} \boldsymbol{g} \cdot \boldsymbol{v} d \boldsymbol{S}-\int_{\Omega} \underset{\approx}{\boldsymbol{\sigma}}\left(\boldsymbol{u}_{X}\right): \underset{\approx}{\boldsymbol{\epsilon}}(\boldsymbol{v}) d \boldsymbol{x} \\
& =\sum_{K \in \mathcal{P}}\left(\int_{K} \boldsymbol{f} \cdot \boldsymbol{v} d \boldsymbol{x}+\sum_{\gamma \in \mathcal{F}_{K} \cap \mathcal{F}_{N}} \int_{\gamma} \boldsymbol{g} \cdot \boldsymbol{v} d \boldsymbol{S}-\int_{K} \underset{\approx}{\boldsymbol{\sigma}}\left(\boldsymbol{u}_{X}\right): \underset{\approx}{\boldsymbol{\epsilon}}(\boldsymbol{v}) d \boldsymbol{x}\right) .
\end{aligned}
$$

3.1. Equilibrated stresses on tetrahedral elements. Let $\mathcal{N}$ index the set $\left\{\boldsymbol{x}_{n}\right\}_{n \in \mathcal{N}}$ of vertices of the elements in $\mathcal{P}$. For $n \in \mathcal{N}$, let $\mathcal{P}_{n}$ denote the set of elements in $\mathcal{P}$ that have a vertex at $\boldsymbol{x}_{n}$ and let $\lambda_{n}$ denote the function which is piecewise linear on $\mathcal{P}$ and vanishes at all the vertices in $\mathcal{P}$, except $\boldsymbol{x}_{n}$, where it takes the value one. Define $\boldsymbol{\lambda}_{n}^{(1)}=\left(\begin{array}{c}\lambda_{n} \\ 0 \\ 0\end{array}\right), \boldsymbol{\lambda}_{n}^{(2)}=\left(\begin{array}{c}0 \\ \lambda_{n} \\ 0\end{array}\right)$ and $\boldsymbol{\lambda}_{n}^{(3)}=\left(\begin{array}{c}0 \\ 0 \\ \lambda_{n}\end{array}\right)$. Also, let $\mathcal{N}(\gamma)$ denote the subset of $\mathcal{N}$ which indexes the three vertices of face $\gamma$.

We want to define equilibrated stress functions $\boldsymbol{g}_{K, \gamma} \in \mathbb{P}_{1}(\gamma)^{3}$ satisfying the conditions

$$
\begin{aligned}
\boldsymbol{g}_{K, \gamma}+\boldsymbol{g}_{K^{\prime}, \gamma} & =\mathbf{0} \text { if } \gamma \in \mathcal{F}_{K} \cap \mathcal{F}_{K^{\prime}}, K, K^{\prime} \in \mathcal{P} \\
\boldsymbol{g}_{K, \gamma} & =P_{\gamma} \boldsymbol{g} \text { if } \gamma \in \mathcal{F}_{N}
\end{aligned}
$$

along with the equilibration condition

$$
\int_{K} \boldsymbol{f} \cdot \boldsymbol{p} d \boldsymbol{x}+\sum_{\gamma \in \mathcal{F}_{K}} \int_{\gamma} \boldsymbol{g}_{K, \gamma} \cdot \boldsymbol{p} d \boldsymbol{S}-\int_{K} \underset{\approx}{\boldsymbol{\sigma}}\left(\boldsymbol{u}_{X}\right): \underset{\approx}{\boldsymbol{\epsilon}}(\boldsymbol{p}) d \boldsymbol{x}=0 \forall \boldsymbol{p} \in \mathbb{P}_{1}(K)^{3}
$$

for all $K \in \mathcal{P}$. We note that conditions (3.2) and (3.3) imply that

$$
\sum_{K \in \mathcal{P}} \sum_{\gamma \in \mathcal{F}_{K} \cap \mathcal{F}_{N}} \int_{\gamma} P_{\gamma} \boldsymbol{g} \cdot \boldsymbol{v} d \boldsymbol{S}=\sum_{K \in \mathcal{P}} \sum_{\gamma \in \mathcal{F}_{K}} \int_{\gamma} \boldsymbol{g}_{K, \gamma} \cdot \boldsymbol{v} d \boldsymbol{S} \forall \boldsymbol{v} \in \boldsymbol{H}_{D}^{1}(\Omega) .
$$

We also note that the values of the nine moments

$$
\mu_{K, n}^{\gamma, i}=\int_{\gamma} \boldsymbol{g}_{K, \gamma} \cdot \boldsymbol{\lambda}_{n}^{(i)} d \boldsymbol{S} \text { for } n \in \mathcal{N}(\gamma) \text { and } i=1,2,3
$$

determine a unique stress $\boldsymbol{g}_{K, \gamma} \in \mathbb{P}_{1}(\gamma)^{3}$. This means that the stress field can be written as a linear combination of the stress moments, but it will not be necessary to actually construct this representation in the code. In fact, the estimator which we shall derive is expressed directly in terms of the moments $\mu_{K, n}^{\gamma, i}$. 
The computational procedure used to determine the moments $\mu_{K, n}^{\gamma, i}$ is virtually identical to the one described in [3] for the case of scalar problems in $\mathbb{R}^{2}$ (with element faces in $\mathbb{R}^{3}$ playing the same role as edges in [3]). For convenience, we briefly outline the main steps.

Let

$$
\left\langle\underset{\sim}{\boldsymbol{g}}\left(\boldsymbol{u}_{\boldsymbol{X}}\right) \boldsymbol{n}_{K}\right\rangle_{\gamma}= \begin{cases}\frac{1}{2}\left(\underset{\sim}{\boldsymbol{g}}\left(\boldsymbol{u}_{\boldsymbol{X} \mid K}\right) \boldsymbol{n}_{\gamma}^{K}-\underset{\sim}{\boldsymbol{g}}\left(\boldsymbol{u}_{\boldsymbol{X} \mid K^{\prime}}\right) \boldsymbol{n}_{\gamma}^{K^{\prime}}\right) & \text { if } \gamma \in \mathcal{F}_{K} \cap \mathcal{F}_{K^{\prime}}, \\ \underset{\widetilde{\boldsymbol{g}}}{\boldsymbol{\sigma}}\left(\boldsymbol{u}_{\boldsymbol{X} \mid K}\right) \boldsymbol{n}_{\gamma}^{K} & \text { if } \gamma \in \mathcal{F}_{K} \cap \mathcal{F}_{D}, \\ \boldsymbol{P}_{\gamma} & \text { if } \gamma \in \mathcal{F}_{K} \cap \mathcal{F}_{N},\end{cases}
$$

where $\boldsymbol{n}_{\gamma}^{K}$ denotes the outward unit normal vector to face $\gamma$ of element $K$. We look for moments $\mu_{K, n}^{\gamma, i}$ of $\boldsymbol{g}_{K, \gamma}$ in the form

$$
\mu_{K, n}^{\gamma, i}= \begin{cases}\frac{1}{2}\left(\xi_{K, n}^{(i)}-\xi_{K^{\prime}, n}^{(i)}\right)+\int_{\gamma}\left\langle\underset{\widetilde{\sigma}}{\boldsymbol{\sigma}}\left(\boldsymbol{u}_{\boldsymbol{X}}\right) \boldsymbol{n}_{K}\right\rangle_{\gamma} \cdot \boldsymbol{\lambda}_{n}^{(i)} d \boldsymbol{S} & \text { if } \gamma \in \mathcal{F}_{K} \cap \mathcal{F}_{K^{\prime}}, \\ \xi_{K, n}^{(i)}+\int_{\gamma}\left(\underset{\widetilde{\sigma}}{\boldsymbol{\sigma}}\left(\boldsymbol{u}_{\boldsymbol{X} \mid K}\right) \boldsymbol{n}_{\gamma}^{K}\right) \cdot \boldsymbol{\lambda}_{n}^{(i)} d \boldsymbol{S} & \text { if } \gamma \in \mathcal{F}_{K} \cap \mathcal{F}_{D}, \\ \int_{\gamma} \boldsymbol{g} \cdot \boldsymbol{\lambda}_{n}^{(i)} d \boldsymbol{S} & \text { if } \gamma \in \mathcal{F}_{K} \cap \mathcal{F}_{N} .\end{cases}
$$

This construction means that $\mu_{K, n}^{\gamma, i}+\mu_{K^{\prime}, n}^{\gamma, i}=0$ for $\gamma \in \mathcal{F}_{K} \cap \mathcal{F}_{K^{\prime}}$, which means that the corresponding stresses $\boldsymbol{g}_{K, \gamma}$ will automatically satisfy (3.2) for all choices of $\xi_{K, n}^{(i)}$. Similarly, (3.3) follows directly from the definition of $\mu_{K, n}^{\gamma, i}$ on $\gamma \in \mathcal{F}_{K} \cap \mathcal{F}_{N}$. We determine the free parameters $\xi_{K, n}^{(i)}$ in (3.7) by solving a system of equations analogous to (6.46) in [3]:

$$
\frac{1}{2} \sum_{K^{\prime} \in \mathcal{P}_{n} \cap \mathcal{P}_{K}}\left(\xi_{K, n}^{(i)}-\xi_{K^{\prime}, n}^{(i)}\right)+\sum_{\gamma \in \mathcal{F}_{K} \cap \mathcal{F}_{D} \cap \mathcal{F}_{n}} \xi_{K, n}^{(i)}=\Delta_{K}\left(\boldsymbol{\lambda}_{n}^{(i)}\right) \text { for all } K \in \mathcal{P}_{n}
$$

where $\mathcal{P}_{K}$ denotes the set of elements that share a face with element $K, \mathcal{F}_{n}$ denotes the set of faces that have a vertex at $\boldsymbol{x}_{n}$ and

$$
\Delta_{K}\left(\boldsymbol{\lambda}_{n}^{(i)}\right)=\int_{K} \underset{\sim}{\boldsymbol{\sigma}}\left(\boldsymbol{u}_{X}\right): \underset{\sim}{\boldsymbol{\epsilon}}\left(\boldsymbol{\lambda}_{n}^{(i)}\right) d \boldsymbol{x}-\int_{K} \boldsymbol{f} \cdot \boldsymbol{\lambda}_{n}^{(i)} d \boldsymbol{x}-\sum_{\gamma \in \mathcal{F}_{K}} \int_{\gamma}\left\langle\underset{\sim}{\boldsymbol{g}}\left(\boldsymbol{u}_{\boldsymbol{X}}\right) \boldsymbol{n}_{K}\right\rangle_{\gamma} \cdot \boldsymbol{\lambda}_{n}^{(i)} d \boldsymbol{S}
$$

The above system consists of $\sharp \mathcal{P}_{n}$ equations for $\sharp \mathcal{P}_{n}$ unknowns, where $\sharp$ denotes cardinality. In general, the linear system fails to have a unique solution. Fortunately, as shown in Lemma 5 in [2], a solution can always be found which depends continuously on the data $\left\{\Delta_{K}\left(\boldsymbol{\lambda}_{n}^{(i)}\right), K \in \mathcal{P}_{n}\right\}$ provided that the following compatibility condition holds:

$$
\sum_{K \in \mathcal{P}_{n}} \Delta_{K}\left(\lambda_{n}^{(i)}\right)=0 \forall n \in \mathcal{N}: \boldsymbol{x}_{n} \notin \bar{\Gamma}_{D}
$$

The fact that this compatibility condition does indeed hold follows at once upon using the definition (3.8) along with (2.3) and the fact that, thanks to (3.6),

$$
\sum_{K \in \mathcal{P}_{n}} \sum_{\gamma \in \mathcal{F}_{K}} \int_{\gamma}\left\langle\underset{\sim}{\boldsymbol{g}}\left(\boldsymbol{u}_{\boldsymbol{X}}\right) \boldsymbol{n}_{K}\right\rangle_{\gamma} \cdot \boldsymbol{\lambda}_{n}^{(i)} d \boldsymbol{S}=\int_{\Gamma_{N}} P_{\gamma} \boldsymbol{g} \cdot \boldsymbol{\lambda}_{n}^{(i)} d \boldsymbol{S}=\int_{\Gamma_{N}} \boldsymbol{g} \cdot \boldsymbol{\lambda}_{n}^{(i)} d \boldsymbol{S}
$$

for all $n \in \mathcal{N}$ such that $\boldsymbol{x}_{n} \notin \bar{\Gamma}_{D}$.

The following result establishes the continuous dependence of the stresses on the data:

THEOREM 3.1. If the stresses $\boldsymbol{g}_{K, \gamma}$ are chosen as described above, then there exists a positive constant $C$, independent of the error $\boldsymbol{e}$ and the size of the elements 
in the mesh, such that

$$
\begin{aligned}
& h_{K}^{1 / 2}\left\|\boldsymbol{g}_{K, \gamma}-\left\langle\underset{\approx}{\boldsymbol{g}}\left(\boldsymbol{u}_{\boldsymbol{X}}\right) \boldsymbol{n}_{K}\right\rangle_{\gamma}\right\|_{L_{2}(\gamma)} \\
\leq & C \sum_{n \in \mathcal{N}(\gamma)}\left(\sum_{K^{\prime} \in \mathcal{P}_{n}}\|\boldsymbol{e}\|_{K^{\prime}}+\sum_{K^{\prime} \in \mathcal{P}_{n}} \operatorname{osc}\left(\boldsymbol{f}, K^{\prime}\right)+\sum_{\gamma^{\prime} \in \mathcal{F}_{n} \cap \mathcal{F}_{N}} \operatorname{osc}\left(\boldsymbol{g}, \gamma^{\prime}\right)\right) .
\end{aligned}
$$

Proof. Standard "bubble function" arguments [3,23] can be used to prove that, for $K \in \mathcal{P}$,

$$
h_{K}\left\|P_{K} \boldsymbol{f}+\operatorname{div} \underset{\approx}{\boldsymbol{g}}(\boldsymbol{u})\right\|_{L_{2}(K)} \leq C\left(\|\boldsymbol{e}\|_{K}+\operatorname{osc}(\boldsymbol{f}, K)\right)
$$

and

$$
\begin{aligned}
& h_{K}^{1 / 2} \sum_{\gamma \in \mathcal{F}_{K} \cap \mathcal{F}_{n}}\left\|\boldsymbol{J}_{\gamma}\right\|_{L_{2}(\gamma)} \\
\leq & C\left(\sum_{K^{\prime} \in \mathcal{P}_{K} \cap \mathcal{P}_{n}}\|\boldsymbol{e}\|_{K^{\prime}}+\sum_{K^{\prime} \in \mathcal{P}_{K} \cap \mathcal{P}_{n}} \operatorname{osc}\left(\boldsymbol{f}, K^{\prime}\right)+\sum_{\gamma \in \mathcal{F}_{K} \cap \mathcal{F}_{N} \cap \mathcal{F}_{n}} \operatorname{osc}(\boldsymbol{g}, \gamma)\right)
\end{aligned}
$$

where

$$
\boldsymbol{J}_{\gamma}= \begin{cases}\frac{1}{2}\left(\underset{\approx}{\boldsymbol{g}}\left(\boldsymbol{u}_{\boldsymbol{X} \mid K}\right) \boldsymbol{n}_{\gamma}^{K}+\underset{\approx}{\boldsymbol{g}}\left(\boldsymbol{u}_{\boldsymbol{X} \mid K^{\prime}}\right) \boldsymbol{n}_{\gamma}^{K^{\prime}}\right) & \text { if } \gamma \in \mathcal{F}_{K} \cap \mathcal{F}_{K^{\prime}}, \\ \underset{\tilde{\mathbf{0}}}{\boldsymbol{\sigma}}\left(\boldsymbol{u}_{\boldsymbol{X} \mid K}\right) \boldsymbol{n}_{\gamma}^{K}-P_{\gamma} \boldsymbol{g} & \text { if } \gamma \in \mathcal{F}_{K} \cap \mathcal{F}_{N}, \\ \text { if } \gamma \in \mathcal{F}_{K} \cap \mathcal{F}_{D} .\end{cases}
$$

Now, integration by parts yields

$$
\begin{aligned}
& \left|\Delta_{K}\left(\boldsymbol{\lambda}_{n}^{(i)}\right)\right| \\
& =\mid \sum_{\gamma \in \mathcal{F}_{K}} \int_{\gamma} \underset{\approx}{\boldsymbol{g}}\left(\boldsymbol{u}_{X \mid K}\right) \boldsymbol{n}_{\gamma}^{K} \cdot \boldsymbol{\lambda}_{n}^{(i)} d \boldsymbol{S}-\sum_{\gamma \in \mathcal{F}_{K}} \int_{\gamma}\left\langle\underset{\approx}{\boldsymbol{g}}\left(\boldsymbol{u}_{\boldsymbol{X}}\right) \boldsymbol{n}_{K}\right\rangle_{\gamma} \cdot \boldsymbol{\lambda}_{n}^{(i)} d \boldsymbol{S} \\
& -\int_{K}\left(P_{K} \boldsymbol{f}+\operatorname{div} \underset{\approx}{\boldsymbol{g}}(\boldsymbol{u})\right) \cdot \boldsymbol{\lambda}_{n}^{(i)} d \boldsymbol{x} \\
& =\left|\sum_{\gamma \in \mathcal{F}_{K}} \int_{\gamma} \boldsymbol{J}_{\gamma} \cdot \boldsymbol{\lambda}_{n}^{(i)} d \boldsymbol{S}-\int_{K}\left(P_{K} \boldsymbol{f}+\operatorname{div} \underset{\approx}{\boldsymbol{\sigma}}(\boldsymbol{u})\right) \cdot \boldsymbol{\lambda}_{n}^{(i)} d \boldsymbol{x}\right| \\
& \leq \sum_{\gamma \in \mathcal{F}_{K}}\left\|\boldsymbol{J}_{\gamma}\right\|_{L_{2}(\gamma)}\left\|\boldsymbol{\lambda}_{n}^{(i)}\right\|_{L_{2}(\gamma)}+\left\|P_{K} \boldsymbol{f}+\operatorname{div} \underset{\approx}{\boldsymbol{\sigma}}(\boldsymbol{u})\right\|_{L_{2}(K)}\left\|\boldsymbol{\lambda}_{n}^{(i)}\right\|_{L_{2}(K)} \\
& \leq C\left(h_{K} \sum_{\gamma \in \mathcal{F}_{K} \cap \mathcal{F}_{n}}\left\|\boldsymbol{J}_{\gamma}\right\|_{L_{2}(\gamma)}+h_{K}^{3 / 2}\left\|P_{K} \boldsymbol{f}+\operatorname{div} \underset{\approx}{\boldsymbol{\sigma}}(\boldsymbol{u})\right\|_{L_{2}(K)}\right) \\
& \leq C h_{K}^{1 / 2}\left(\sum_{K^{\prime} \in \mathcal{P}_{K} \cap \mathcal{P}_{n}}\|\boldsymbol{e}\|_{K^{\prime}}+\sum_{K^{\prime} \in \mathcal{P}_{K} \cap \mathcal{P}_{n}} \operatorname{osc}\left(\boldsymbol{f}, K^{\prime}\right)+\sum_{\gamma \in \mathcal{F}_{K} \cap \mathcal{F}_{N} \cap \mathcal{F}_{n}} \operatorname{osc}(\boldsymbol{g}, \gamma)\right)
\end{aligned}
$$

upon inserting bounds (3.10) and (3.11). Finally, (33) from [2] means that

$$
h_{K}^{-1 / 2}\left|\int_{\gamma}\left(\boldsymbol{g}_{K, \gamma}-\left\langle\underset{\approx}{\boldsymbol{g}}\left(\boldsymbol{u}_{\boldsymbol{X}}\right) \boldsymbol{n}_{K}\right\rangle_{\gamma}\right) \cdot \boldsymbol{\lambda}_{n}^{(i)} d \boldsymbol{S}\right| \leq C h_{K}^{-1 / 2} \sum_{K^{\prime} \in \mathcal{P}_{n}}\left|\Delta_{K^{\prime}}\left(\boldsymbol{\lambda}_{n}^{(i)}\right)\right|
$$


from which it follows that

$$
\begin{aligned}
& h_{K}^{-1 / 2}\left|\int_{\gamma}\left(\boldsymbol{g}_{K, \gamma}-\left\langle\underset{\approx}{\boldsymbol{g}}\left(\boldsymbol{u}_{\boldsymbol{X}}\right) \boldsymbol{n}_{K}\right\rangle_{\gamma}\right) \cdot \boldsymbol{\lambda}_{n}^{(i)} d \boldsymbol{S}\right| \\
\leq & C\left(\sum_{K^{\prime} \in \mathcal{P}_{n}}\|\boldsymbol{e}\|_{K^{\prime}}+\sum_{K^{\prime} \in \mathcal{P}_{n}} \operatorname{osc}\left(\boldsymbol{f}, K^{\prime}\right)+\sum_{\gamma^{\prime} \in \mathcal{F}_{n} \cap \mathcal{F}_{N}} \operatorname{osc}\left(\boldsymbol{g}, \gamma^{\prime}\right)\right)
\end{aligned}
$$

upon combining the above bounds. Inequality (3.9) is then a direct consequence of (3.13) and the fact that $\boldsymbol{g}_{K, \gamma}$ is affine. $\square$

3.2. Upper bounds on the energy norm of the error. Substituting (3.5) into (3.1) gives

$$
\begin{aligned}
\int_{\Omega} \underset{\approx}{\boldsymbol{\sigma}}(\boldsymbol{e}): \underset{\approx}{\boldsymbol{\epsilon}}(\boldsymbol{v}) d \boldsymbol{x}= & \sum_{K \in \mathcal{P}}\left(\int_{K} \boldsymbol{f} \cdot \boldsymbol{v} d \boldsymbol{x}+\sum_{\gamma \in \mathcal{F}_{K}} \int_{\gamma} \boldsymbol{g}_{K, \gamma} \cdot \boldsymbol{v} d \boldsymbol{S}\right. \\
& \left.-\int_{K} \underset{\approx}{\boldsymbol{\sigma}}\left(\boldsymbol{u}_{X}\right): \underset{\approx}{\boldsymbol{\epsilon}}(\boldsymbol{v}) d \boldsymbol{x}\right) .
\end{aligned}
$$

Now, integrating by parts yields

$$
\begin{aligned}
\int_{\Omega} \underset{\approx}{\boldsymbol{\sigma}}(\boldsymbol{e}): \underset{\approx}{\boldsymbol{\epsilon}}(\boldsymbol{v}) d \boldsymbol{x}= & \sum_{K \in \mathcal{P}}\left(\int_{K} \boldsymbol{f} \cdot \boldsymbol{v} d \boldsymbol{x}+\sum_{\gamma \in \mathcal{F}_{K}} \int_{\gamma} \boldsymbol{g}_{K, \gamma} \cdot \boldsymbol{v} d \boldsymbol{S}\right. \\
& \left.-\sum_{\gamma \in \mathcal{F}_{K}} \int_{\gamma}\left(\underset{\approx}{\boldsymbol{\sigma}}\left(\boldsymbol{u}_{X \mid K}\right) \boldsymbol{n}_{\gamma}^{K}\right) \cdot \boldsymbol{v} d \boldsymbol{S}+\int_{K}\left(\operatorname{div} \underset{\approx}{\boldsymbol{\sigma}}\left(\boldsymbol{u}_{X}\right)\right) \cdot \boldsymbol{v} d \boldsymbol{x}\right) .
\end{aligned}
$$

The above expression can then be rewritten in the form

$$
\begin{aligned}
\int_{\Omega} \underset{\approx}{\boldsymbol{\sigma}}(\boldsymbol{e}): \underset{\approx}{\boldsymbol{\epsilon}}(\boldsymbol{v}) d \boldsymbol{x}= & \sum_{K \in \mathcal{P}}\left(\int_{K} \boldsymbol{r}_{K} \cdot \boldsymbol{v} d \boldsymbol{x}+\sum_{\gamma \in \mathcal{F}_{K}} \int_{\gamma} \boldsymbol{R}_{\gamma, K} \cdot \boldsymbol{v} d \boldsymbol{S}\right) \\
& +\sum_{K \in \mathcal{P}} \int_{K}\left(\boldsymbol{f}-P_{K} \boldsymbol{f}\right) \cdot \boldsymbol{v} d \boldsymbol{x}+\sum_{\gamma \in \mathcal{F}_{N}} \int_{\gamma}\left(\boldsymbol{g}-P_{\gamma} \boldsymbol{g}\right) \cdot \boldsymbol{v} d \boldsymbol{S}
\end{aligned}
$$

where the interior residual $\boldsymbol{r}_{K} \in \mathbb{P}_{1}(K)^{3}$ is defined by

$$
\boldsymbol{r}_{K}=P_{K} \boldsymbol{f}+\operatorname{div} \underset{\approx}{\boldsymbol{g}}\left(\boldsymbol{u}_{X \mid K}\right) \text { on } K
$$

and the boundary residual $\boldsymbol{R}_{\gamma, K} \in \mathbb{P}_{1}(\gamma)^{3}$ is defined by

$$
\boldsymbol{R}_{\gamma, K}=\boldsymbol{g}_{K, \gamma}-\underset{\approx}{\boldsymbol{g}}\left(\boldsymbol{u}_{X \mid K}\right) \boldsymbol{n}_{\gamma}^{K} \text { on } \gamma
$$

Since the stresses $\boldsymbol{g}_{K, \gamma}$ have been chosen such that (3.4) holds, then these residuals satisfy

$$
\sum_{\gamma \in \mathcal{F}_{K}} \int_{\gamma} \boldsymbol{R}_{\gamma, K} \cdot \boldsymbol{p} d \boldsymbol{S}+\int_{K} \boldsymbol{r}_{K} \cdot \boldsymbol{p} d \boldsymbol{x}=0 \text { for all } \boldsymbol{p} \in \mathbb{P}_{1}(K)^{3}
$$

For future reference, we note that choosing the stresses $\boldsymbol{g}_{K, \gamma}$ in the standard way described above does not enforce any relationship between the $\boldsymbol{R}_{\gamma, K}$ beyond (3.16) and so, in general,

$$
\boldsymbol{n}_{\gamma^{\prime}}^{K} \cdot \boldsymbol{R}_{\gamma, K} \neq \boldsymbol{n}_{\gamma}^{K} \cdot \boldsymbol{R}_{\gamma^{\prime}, K}
$$


on the edge shared by faces $\gamma$ and $\gamma^{\prime}$ of element $K$.

We define the set of symmetric tensors in $\underset{\sim}{\boldsymbol{H}}(\operatorname{div} ; K)$ by

$$
\underset{\approx}{\boldsymbol{H}}{ }^{\mathrm{Sym}}(\operatorname{div} ; K)=\left\{\underset{\approx}{\boldsymbol{v}} \in \underset{\approx}{\boldsymbol{H}}(\operatorname{div} ; K): \boldsymbol{\alpha}_{1}^{T} \underset{\approx}{\boldsymbol{v}} \boldsymbol{\alpha}_{2}=\boldsymbol{\alpha}_{2}^{T} \underset{\approx}{\boldsymbol{v}} \boldsymbol{\alpha}_{1} \text { for all } \boldsymbol{\alpha}_{1}, \boldsymbol{\alpha}_{2} \in \mathbb{R}^{3}\right\}
$$

and the associated subspace

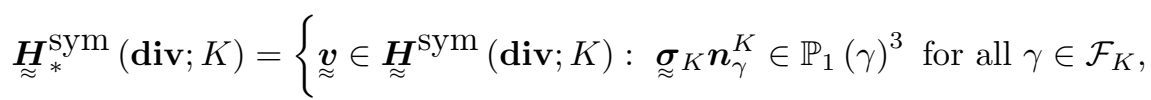

$$
\begin{aligned}
& \left.\operatorname{div} \underset{\approx}{\boldsymbol{v}} \in \mathbb{P}_{1}(K)^{3}, \int_{K} \underset{\approx}{\boldsymbol{v}}: \underset{\approx}{\boldsymbol{\epsilon}}(\boldsymbol{p}) d \boldsymbol{x}=0 \text { for all } \boldsymbol{p} \in \mathbb{P}_{1}(K)^{3}\right\}
\end{aligned}
$$

Observe that both spaces are infinite dimensional.

We can now state our main result giving a computable upper bound on the energy norm of the error $\boldsymbol{e}$ in the finite element approximation:

THEOREM 3.2. Suppose $\underset{\approx}{\boldsymbol{\sigma}_{K}} \in \underset{\sim}{\boldsymbol{H}_{*}^{\text {sym }}}$ (div; $\left.K\right)$ satisfies the condition

$$
\underset{\approx}{\boldsymbol{\sigma}}{ }_{K} \boldsymbol{n}_{\gamma}^{K}=\boldsymbol{R}_{\gamma, K} \text { on } \gamma \text { for all } \gamma \in \mathcal{F}_{K}
$$

Then

$$
\int_{K} \underset{\approx}{\boldsymbol{\sigma}}{ }_{K}: \underset{\approx}{\boldsymbol{\epsilon}}(\boldsymbol{v}) d \boldsymbol{x}=\sum_{\gamma \in \mathcal{F}_{K}} \int_{\gamma} \boldsymbol{R}_{\gamma, K} \cdot \boldsymbol{v} d \boldsymbol{S}+\int_{K} \boldsymbol{r}_{K} \cdot \boldsymbol{v} d \boldsymbol{x} \text { for all } \boldsymbol{v} \in \boldsymbol{H}^{1}(\Omega)
$$

and

$$
\|\boldsymbol{e}\| \leq\left(\sum_{K \in \mathcal{P}} \int_{K} \mathbb{E}^{-1}{\underset{\approx}{\boldsymbol{\sigma}}}_{K}:{\underset{\approx}{\boldsymbol{\sigma}} K}_{K} d \boldsymbol{x}\right)^{1 / 2}+\frac{C_{\Omega}}{\sqrt{2 \mu}}\left(\sum_{K \in \mathcal{P}} \Psi_{K}^{2}\right)^{1 / 2}
$$

where $C_{\Omega}$ is the constant in (2.2) and

$$
\Psi_{K}=\frac{1}{\pi} \operatorname{osc}(\boldsymbol{f}, K)+\sum_{\gamma \in \mathcal{F}_{K} \cap \mathcal{F}_{N}} C_{\gamma}^{K} \operatorname{osc}(\boldsymbol{g}, \gamma)
$$

with

$$
C_{\gamma}^{K}=\left(\frac{|\gamma|^{1 / 2}}{|K|} \frac{h_{K}}{\pi}\left(\frac{h_{K}}{\pi}+\frac{2}{3} \max _{\boldsymbol{x} \in \gamma}\left|\boldsymbol{x}-\boldsymbol{x}_{\gamma}\right|\right)\right)^{1 / 2}
$$

where $h_{K}$ is the length of the longest edge of element $K$ and $\boldsymbol{x}_{\gamma}$ is the vertex of element $K$ which is not a vertex of face $\gamma$. Details on how to compute the value of $\int_{K} \mathbb{E}^{-1}{\underset{\approx}{\boldsymbol{\sigma}}}_{K}: \underset{\approx}{\boldsymbol{\sigma}_{K}} d \boldsymbol{x}$ directly without having to construct $\underset{\approx}{\boldsymbol{\sigma}_{K}}$ explicitly are given in Section 4.

Proof. For $\underset{\approx}{\boldsymbol{\sigma}_{K} \in \underset{\sim}{\boldsymbol{H}_{*}}}$ Sym $(\operatorname{div} ; K)$, integration by parts yields

$$
\begin{aligned}
\int_{K} \underset{\approx}{\boldsymbol{\sigma}}{ }_{K}: \underset{\approx}{\boldsymbol{\epsilon}}(\boldsymbol{v}) d \boldsymbol{x} & =\sum_{\gamma \in \mathcal{F}_{K}} \int_{\gamma}\left(\underset{\approx}{\boldsymbol{\sigma}_{K}} \boldsymbol{n}_{\gamma}^{K}\right) \cdot \boldsymbol{v} d \boldsymbol{S}-\int_{K}(\operatorname{div} \underset{\approx}{\boldsymbol{\sigma}}) \cdot \boldsymbol{v} d \boldsymbol{x} \\
& =\sum_{\gamma \in \mathcal{F}_{K}} \int_{\gamma} \boldsymbol{R}_{\gamma, K} \cdot \boldsymbol{v} d \boldsymbol{S}-\int_{K}\left(\operatorname{div} \underset{\approx}{\boldsymbol{\sigma}_{K}}\right) \cdot \boldsymbol{v} d \boldsymbol{x}
\end{aligned}
$$

upon inserting (3.18). By letting $\boldsymbol{v}=\boldsymbol{p} \in \mathbb{P}_{1}(K)^{3}$ in (3.23) we can see that

$$
\sum_{\gamma \in \mathcal{F}_{K}} \int_{\gamma} \boldsymbol{R}_{\gamma, K} \cdot \boldsymbol{p} d \boldsymbol{S}-\int_{K}(\operatorname{div} \underset{\approx}{\boldsymbol{\sigma}} K) \cdot \boldsymbol{p} d \boldsymbol{x}=0 \text { for all } \boldsymbol{p} \in \mathbb{P}_{1}(K)^{3}
$$


by the definition of $\underset{\approx}{\boldsymbol{H}}$ Sym $(\operatorname{div} ; K)$. From (3.16) it follows that

$$
\int_{K}\left(\boldsymbol{r}_{K}+\operatorname{div}{\underset{\approx}{\boldsymbol{g}}}_{K}\right) \cdot \boldsymbol{p} d \boldsymbol{x}=0 \text { for all } p \in \mathbb{P}_{1}(K)^{3}
$$

which means that

$$
-\operatorname{div} \underset{\approx}{\boldsymbol{\sigma}_{K}}=\boldsymbol{r}_{K} \text { in } K
$$

since both $\boldsymbol{r}_{K}$ and $\operatorname{div} \underset{\approx}{\boldsymbol{\sigma}} K$ belong to $\mathbb{P}_{1}(K)^{3}$ by the definition of $\underset{\approx}{\boldsymbol{H}}$ sym $(\operatorname{div} ; K)$. Finally, substituting (3.2) into (3.23) yields (3.19).

We can now return to $(3.14)$ and replace the first two terms on the right hand side using (3.19) and then use the estimates

$$
\int_{K}\left(\boldsymbol{f}-P_{K} \boldsymbol{f}\right) \cdot \boldsymbol{v} d \boldsymbol{x} \leq \frac{1}{\pi} \operatorname{osc}(\boldsymbol{f}, K)\left(\int_{K} \underset{\approx}{\operatorname{grad} \boldsymbol{v}}: \underset{\approx}{\operatorname{grad} \boldsymbol{v}} d \boldsymbol{x}\right)^{1 / 2}
$$

and

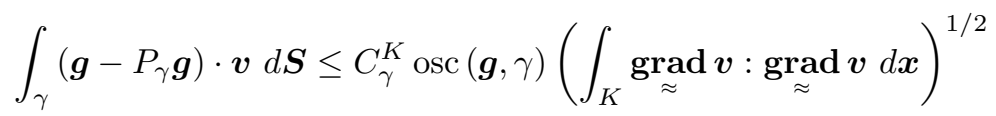

(which we prove in Lemma 3.7) to conclude that

$$
\begin{aligned}
& \int_{\Omega} \underset{\approx}{\boldsymbol{\sigma}}(\boldsymbol{e}): \underset{\approx}{\boldsymbol{\epsilon}}(\boldsymbol{v}) d \boldsymbol{x} \\
\leq & \sum_{K \in \mathcal{P}}\left(\int_{K} \underset{\approx}{\boldsymbol{\sigma}}{ }_{K}: \underset{\approx}{\boldsymbol{\epsilon}}(\boldsymbol{v}) d \boldsymbol{x}+\Psi_{K}\left(\int_{K} \underset{\approx}{\operatorname{grad} \boldsymbol{v}}: \underset{\approx}{\operatorname{grad}} \boldsymbol{v} d \boldsymbol{x}\right)^{1 / 2}\right) .
\end{aligned}
$$

Let

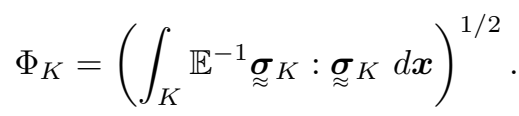

Then using the Cauchy-Schwarz inequality

$$
\int_{K} \underset{\approx}{\boldsymbol{\sigma}_{K}}: \underset{\approx}{\boldsymbol{\epsilon}}(\boldsymbol{v}) d \boldsymbol{x} \leq\left(\int_{K} \mathbb{E}^{-1}{\underset{\approx}{\boldsymbol{g}}}_{K}:{\underset{\approx}{\boldsymbol{g}}}_{K} d \boldsymbol{x}\right)^{1 / 2}\|\boldsymbol{v}\|_{K}=\Phi_{K}\|\boldsymbol{v}\|_{K},
$$

we obtain the bounds

$$
\begin{aligned}
& \int_{\Omega} \underset{\approx}{\boldsymbol{\sigma}}(\boldsymbol{e}): \underset{\approx}{\boldsymbol{\epsilon}}(\boldsymbol{v}) d \boldsymbol{x} \\
& \leq \sum_{K \in \mathcal{P}} \Phi_{K}\|\boldsymbol{v}\|_{K}+\sum_{K \in \mathcal{P}} \Psi_{K}\left(\int_{K} \underset{\approx}{\operatorname{grad}} \boldsymbol{v}: \underset{\approx}{\operatorname{grad}} \boldsymbol{v} d \boldsymbol{x}\right)^{1 / 2}
\end{aligned}
$$

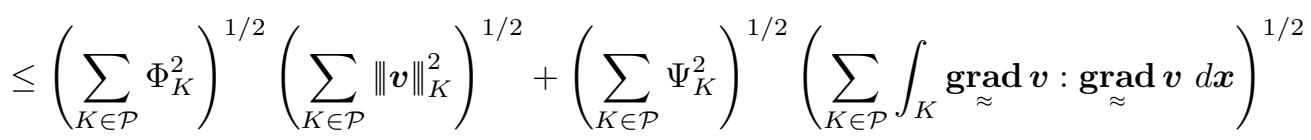

$$
\begin{aligned}
& =\left(\sum_{K \in \mathcal{P}} \Phi_{K}^{2}\right)^{1 / 2}\|\boldsymbol{v}\|+\left(\sum_{K \in \mathcal{P}} \Psi_{K}^{2}\right)^{1 / 2}\left(\int_{\Omega} \underset{\approx}{\operatorname{grad}} \boldsymbol{v}: \underset{\approx}{\operatorname{grad}} \boldsymbol{v} d \boldsymbol{x}\right)^{1 / 2} .
\end{aligned}
$$

We can then apply (2.2) and make use of the fact that

$$
\left(\int_{K} \underset{\approx}{\boldsymbol{\epsilon}}(\boldsymbol{v}): \underset{\approx}{\boldsymbol{\epsilon}}(\boldsymbol{v}) d \boldsymbol{x}\right)^{1 / 2} \leq \frac{1}{\sqrt{2 \mu}}\|\boldsymbol{v}\|_{K}
$$


to conclude that

$$
\int_{\Omega} \underset{\approx}{\boldsymbol{\sigma}}(\boldsymbol{e}): \underset{\approx}{\boldsymbol{\epsilon}}(\boldsymbol{v}) d \boldsymbol{x} \leq\left(\left(\sum_{K \in \mathcal{P}} \Phi_{K}^{2}\right)^{1 / 2}+\frac{C_{\Omega}}{\sqrt{2 \mu}}\left(\sum_{K \in \mathcal{P}} \Psi_{K}^{2}\right)^{1 / 2}\right)\|\boldsymbol{v}\|
$$

Finally, letting $\boldsymbol{v}=\boldsymbol{e}$ in the above expression and dividing through by $\|\boldsymbol{e}\|$ we arrive at (3.20) in the case when $\|\boldsymbol{e}\| \neq 0$. In the case when $\|\boldsymbol{e}\|=0$ the bound holds trivially.

It is also possible to obtain an alternative bound:

COROLlaRY 3.3. Suppose $\underset{\approx}{\boldsymbol{\sigma}_{K}} \in \underset{\approx}{\boldsymbol{H}_{*}^{\text {sym }}}$ (div; $K$ ) satisfies (3.18). Then

$$
\|\boldsymbol{e}\| \leq\left(\sum_{K \in \mathcal{P}}\left(\left(\int_{K} \mathbb{E}^{-1}{\underset{\approx}{\boldsymbol{\sigma}}}_{K}:{\underset{\approx}{\boldsymbol{g}_{K}}} d \boldsymbol{x}\right)^{1 / 2}+\frac{C_{K}}{\sqrt{2 \mu}} \Psi_{K}\right)^{2}\right)^{1 / 2}
$$

where $C_{K}$ denotes the constant in the local Korn's inequality

$$
\inf _{\boldsymbol{v}_{K} \in \mathcal{R}(K)}\left(\int_{K} \underset{\approx}{\operatorname{grad}}\left(\boldsymbol{v}-\boldsymbol{v}_{K}\right): \underset{\approx}{\operatorname{grad}}\left(\boldsymbol{v}-\boldsymbol{v}_{K}\right) d \boldsymbol{x}\right)^{1 / 2} \leq C_{K}\left(\int_{K} \underset{\approx}{\boldsymbol{\epsilon}}(\boldsymbol{v}): \underset{\approx}{\boldsymbol{\epsilon}}(\boldsymbol{v}) d \boldsymbol{x}\right)^{1 / 2}
$$

for all $\boldsymbol{v} \in \boldsymbol{H}_{D}^{1}(K)=\left\{\boldsymbol{v} \in \boldsymbol{H}^{1}(K): \boldsymbol{v}=\mathbf{0}\right.$ on $\left.\Gamma_{D}\right\}$ where

$$
\mathcal{R}(K)=\left\{\boldsymbol{v} \in \boldsymbol{H}^{1}(K): \underset{\approx}{\boldsymbol{\epsilon}}(\boldsymbol{v})=\underset{\approx}{\mathbf{0}}\right\} \subset \mathbb{P}_{1}(K)^{3} .
$$

Proof. In the proof of Theorem 3.2 we can use the estimates

$$
\int_{K}\left(\boldsymbol{f}-P_{K} \boldsymbol{f}\right) \cdot \boldsymbol{v} d \boldsymbol{x} \leq \frac{C_{K}}{\pi} \operatorname{osc}(\boldsymbol{f}, K)\left(\int_{K} \underset{\approx}{\boldsymbol{\epsilon}}(\boldsymbol{v}): \underset{\approx}{\boldsymbol{\epsilon}}(\boldsymbol{v}) d \boldsymbol{x}\right)^{1 / 2}
$$

and

$$
\int_{\gamma}\left(\boldsymbol{g}-P_{\gamma} \boldsymbol{g}\right) \cdot \boldsymbol{v} d \boldsymbol{S} \leq C_{K} C_{\gamma}^{K} \operatorname{osc}(\boldsymbol{g}, \gamma)\left(\int_{K} \underset{\approx}{\boldsymbol{\epsilon}}(\boldsymbol{v}): \underset{\approx}{\boldsymbol{\epsilon}}(\boldsymbol{v}) d \boldsymbol{x}\right)^{1 / 2}
$$

(which we prove in Lemma 3.8) instead of (3.25) and (3.26) to arrive at

$$
\begin{aligned}
\int_{\Omega} \underset{\approx}{\boldsymbol{\sigma}}(\boldsymbol{e}): \underset{\approx}{\boldsymbol{\epsilon}}(\boldsymbol{v}) d \boldsymbol{x} & \leq \sum_{K \in \mathcal{P}} \Phi_{K}\|\boldsymbol{v}\|_{K}+C_{K} \Psi_{K}\left(\int_{K} \underset{\approx}{\boldsymbol{\epsilon}}(\boldsymbol{v}): \underset{\sim}{\boldsymbol{\epsilon}}(\boldsymbol{v}) d \boldsymbol{x}\right)^{1 / 2} \\
& \leq \sum_{K \in \mathcal{P}}\left(\Phi_{K}+\frac{C_{K}}{\sqrt{2 \mu}} \Psi_{K}\right)\|\boldsymbol{v}\|_{K} \\
& \leq\left(\sum_{K \in \mathcal{P}}\left(\Phi_{K}+\frac{C_{K}}{\sqrt{2 \mu}} \Psi_{K}\right)^{2}\right)^{1 / 2}\|\boldsymbol{v}\|
\end{aligned}
$$

from which we obtain $(3.27)$.

An upper bound on the constant $C_{K}$ in the two dimensional analog of (3.28) is given in [14]. However, as far as we are aware, an upper bound for the constant $C_{K}$ in (3.28) is not known in three dimensions. In many practical applications of finite elements, one sees the engineer choosing data $f \in \mathbb{P}_{1}(K)^{3}$ on each element $K \in \mathcal{P}$ and $\boldsymbol{g} \in \mathbb{P}_{1}(\gamma)^{3}$ on each face $\gamma \in \mathcal{F}_{N}$ (or even piecewise constant), in which case the oscillation terms are absent and knowledge of $C_{K}$ or $C_{\Omega}$ becomes a moot point. 
3.3. Construction of a stress representer. Throughout this section we shall only be considering a single element $K$, and hence, for ease of notation we omit the superscript from $\boldsymbol{n}_{\gamma}^{K}$. Our computable upper bound hinges on the existence (and construction) of a suitable stress representer $\underset{\approx}{\boldsymbol{\sigma}} \in \underset{\approx}{\boldsymbol{\sim}}$ Sym $(\mathbf{d i v} ; K)$ satisfying (3.18).

THEOREM 3.4. There exists $\underset{\sim}{\boldsymbol{\sigma}}{ }_{K} \in \underset{\sim}{\boldsymbol{H}}{ }_{*}^{\text {sym }}(\mathbf{d i v} ; K)$ satisfying (3.18).

Proof. In order to prove the existence of $\underset{\widetilde{\sigma}}{\boldsymbol{\sigma}_{K}}$, we consider the related problem of seeking a displacement $\phi_{K} \in \mathcal{H}(K)=\boldsymbol{H}^{1}(K) \backslash \mathcal{R}(K)$ satisfying

$$
\int_{K} \underset{\approx}{\boldsymbol{\sigma}}\left(\phi_{K}\right): \underset{\approx}{\boldsymbol{\epsilon}}(\boldsymbol{v}) d \boldsymbol{x}=\int_{K} \boldsymbol{r}_{K} \cdot \boldsymbol{v} d \boldsymbol{x}+\sum_{\gamma \in \mathcal{F}_{K}} \int_{\gamma} \boldsymbol{R}_{\gamma, K} \cdot \boldsymbol{v} d \boldsymbol{S} \forall \boldsymbol{v} \in \mathcal{H}(K) .
$$

The bilinear form on the left hand side of (3.30) is trivially seen to be coercive on $\mathcal{H}(K)$ whilst the linear form on the right hand side of $(3.30)$ is continuous on $\mathcal{H}(K)$. Hence, the Lax-Milgram Lemma ensures the existence of a unique solution $\phi_{K}$. Let $\underset{\approx}{\boldsymbol{\sigma}}=\underset{\approx}{\boldsymbol{g}}\left(\phi_{K}\right)$. Then $\underset{\approx}{\boldsymbol{\sigma}} K_{\text {satisfies }}$

$$
\int_{K} \underset{\approx}{\boldsymbol{\sigma}_{K}}: \underset{\approx}{\boldsymbol{\epsilon}}(\boldsymbol{v}) d \boldsymbol{x}=\int_{K} \boldsymbol{r}_{K} \cdot \boldsymbol{v} d \boldsymbol{x}+\sum_{\gamma \in \mathcal{F}_{K}} \int_{\gamma} \boldsymbol{R}_{\gamma, K} \cdot \boldsymbol{v} d \boldsymbol{S} \forall \boldsymbol{v} \in \boldsymbol{H}^{1}(K)
$$

since both sides of (3.31) vanish when $\boldsymbol{v} \in \mathcal{R}(K)$ thanks to (3.16). Consequently, since $\boldsymbol{r}_{K} \in \mathbb{P}_{1}(K)^{3}$ and $\boldsymbol{R}_{\gamma, K} \in \mathbb{P}_{1}(\gamma)^{3}$, identity (3.31) means that $-\operatorname{div} \underset{\approx}{\boldsymbol{\sigma}_{K}}=\boldsymbol{r}_{K}$ in $K$ and $\underset{\approx}{\boldsymbol{g}}{ }_{K} \boldsymbol{n}_{\gamma}=\boldsymbol{R}_{\gamma, K}$ on all $\gamma \in \mathcal{F}_{K}$. Moreover, $\underset{\approx}{\boldsymbol{\sigma}} K \in \underset{\sim}{\boldsymbol{H}}{ }_{*}^{\mathrm{sym}}(\boldsymbol{d i v} ; K)$ since, again thanks to (3.16), (3.31) means that $\int_{K} \underset{\approx}{\boldsymbol{\sigma}_{K}}: \underset{\approx}{\boldsymbol{\epsilon}}(\boldsymbol{v}) d \boldsymbol{x}=0$ when $\boldsymbol{v} \in \mathbb{P}_{1}(K)^{3}$. 口

Theorem 3.4 ensures the existence of a suitable field $\underset{\boldsymbol{\sigma}_{K}}{ }$, satisfying the hypothesis of Theorem 3.2. It is easy to see that $\underset{\widetilde{\sigma}}{\boldsymbol{\sigma}_{K}}$ is non-unique which is indeed fortunate since the construction of $\boldsymbol{\sigma}_{K}$ suggested in the proof of Theorem 3.4 is not a viable practical proposition: (3.30) entails the existence of an infinite dimensional local boundary value problem which cannot be solved analytically in general. Nevertheless, some authors $[8,13,19,20]$ have sought to construct an approximation to $\boldsymbol{\sigma}_{K}$ by approximating (3.30) over a finite dimensional subspace of $\boldsymbol{H}^{1}(K)$. This means that the approximate displacement $\phi_{K}$ fails to satisfy (3.31) over the full space $\boldsymbol{H}^{1}(K)$ meaning that the resulting estimator is no longer a guaranteed upper bound on the error. For this reason, we adopt an alternative approach whereby ${\underset{\widetilde{\sigma}}{K}}_{K}$ is treated directly (i.e. need not be defined in terms of a displacement field $\phi_{K} \tilde{\widetilde{ }}$.

Ideally, we would like to choose a simple direct construction for $\boldsymbol{\sigma}_{K}$ in terms of

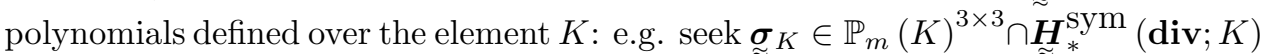
for a suitable $m \in \mathbb{N}$. Unfortunately, this choice is doomed to failure, regardless of the value of $m$, for the following reason. Let $\boldsymbol{\sigma}_{K} \in \mathbb{P}_{m}(K)^{3 \times 3} \cap \boldsymbol{\sim}_{*}^{\text {Sym }}(\operatorname{div} ; K)$, and consider only the edge shared by distinct faces $\gamma_{4}, \gamma_{1} \in \mathcal{F}_{K}$ shown in Figure 3.1. Since, $\underset{\approx}{\boldsymbol{\sigma}}$ is symmetric $\boldsymbol{n}_{\gamma_{4}}^{T} \boldsymbol{\sigma}_{K} \boldsymbol{n}_{\gamma_{1}}=\boldsymbol{n}_{\gamma_{1}}^{T} \boldsymbol{\sigma}_{K} \boldsymbol{n}_{\gamma_{4}}$ on the edge shared by faces $\gamma_{4}$ and $\gamma_{1}$. This means that, if $\underset{\sim}{\boldsymbol{\sigma}_{K}} \in \mathbb{P}_{m}(K)^{3 \times 3} \cap \underset{\widetilde{3}}{\boldsymbol{H}_{*}^{\text {sym }}}(\mathbf{d i v} ; K)$, then (3.18) can only be satisfied if $\boldsymbol{n}_{\gamma_{4}}^{T} \boldsymbol{R}_{\gamma_{1}, K}=\boldsymbol{n}_{\gamma_{1}}^{T} \boldsymbol{R}_{\gamma_{4}, K}$ on the edge shared by faces $\gamma_{4}$ and $\gamma_{1}$ which, as noted earlier in (3.17), is not the case in general.

The above argument shows that one cannot hope to find a polynomial field $\underset{\approx}{\boldsymbol{\sigma}_{K}} \in$ $\underset{\widetilde{\sim}}{\boldsymbol{H}}$ Sym $(\mathbf{d i v} ; K)$ on element $K$ satisfying (3.18). Prompted by our investigations [4] $\underset{\tilde{\sim}}{\tilde{n}}$ the case of planar elasticity we construct a piecewise polynomial field $\boldsymbol{\sigma}_{K}$ over the element as follows. We construct a sub-mesh of four tetrahedra, denoted by $K_{1}, K_{2}$, $K_{3}$ and $K_{4}$, each having a vertex at the centroid of element $K$ and such that $K_{m}$ has $\gamma_{m}$ as one of its faces as shown in Figure 3.2. We then seek $\underset{\approx}{\boldsymbol{\sigma}} K \in \underset{\widetilde{\sigma}_{*}^{\text {Sym }}}{\mathrm{Sym}}(\mathbf{d i v} ; K)$ such that $\underset{\approx}{\boldsymbol{\sigma}_{K \mid K_{m}}} \in \mathbb{P}_{2}\left(K_{m}\right)^{3 \times 3}$ for each sub-tetrahedron $K_{m} \subset K$. In other words, 


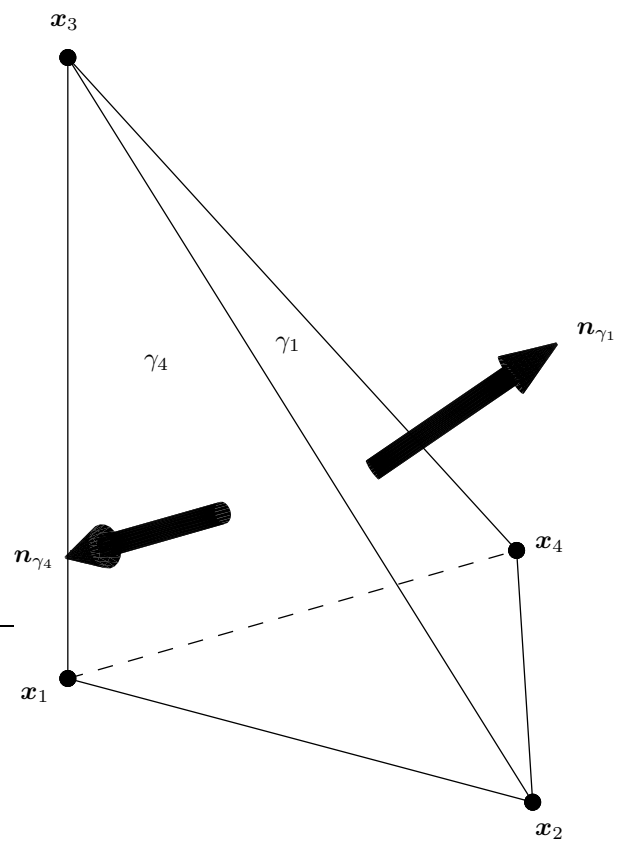

FIG. 3.1. The labelling and positioning of the vertices, faces and unit normal vectors of element $K$. The face $\gamma_{i}$ of element $K$ lies opposite vertex $\boldsymbol{x}_{i}$ and $\boldsymbol{n}_{\gamma_{i}}$ is the outer unit normal vector to face $\gamma_{i}$, for $i=1,2,3,4$.

we seek $\underset{\sim}{\boldsymbol{\sigma}_{K}}$ in the space $\underset{\sim}{\boldsymbol{V}} K$ defined by

$$
\underset{\sim}{\boldsymbol{V}}=\left\{\underset{\sim}{\boldsymbol{v}}: \underset{\sim}{\boldsymbol{v}} \in \underset{\approx}{\boldsymbol{H}}{ }_{*}^{\mathrm{sym}}(\operatorname{div} ; K), \underset{\approx}{\boldsymbol{v}} \mid K_{m} \in \mathbb{P}_{2}\left(K_{m}\right)^{3 \times 3} \text { for } m=1,2,3,4\right\} .
$$

We note that the space $\underset{\sim}{\boldsymbol{V}_{K}}$ is a subspace of the three dimensional analog of the lowest order Arnold, Doüglas and Gupta finite element space [5]. The following result asserts that it is possible to find a ${\underset{\sim}{\sigma}}_{K} \in{\underset{\widetilde{V}}{\boldsymbol{V}}}_{K}$ which satisfies (3.18).

THEOREM 3.5. There exists ${\underset{\sim}{\boldsymbol{\sigma}}}_{K} \in \underset{\sim}{\underset{\sim}{\boldsymbol{\sigma}}}{ }_{K}$ such that

$$
{\underset{\sim}{\sigma}}_{K} \boldsymbol{n}_{\gamma}=\boldsymbol{R}_{\gamma, K} \text { for all } \gamma \in \mathcal{F}_{K}
$$

and there exists a positive constant $C$, independent of the error $\boldsymbol{e}$ and the size of the elements in the mesh, such that

$$
\begin{aligned}
& \left(\int_{K} \mathbb{E}^{-1} \boldsymbol{\sigma}_{\boldsymbol{\sim}}:{\underset{\sim}{\boldsymbol{\sigma}_{K}}}_{K} d \boldsymbol{x}\right)^{1 / 2} \\
\leq & C\left(\sum_{K^{\prime} \in \tilde{K}}\|\boldsymbol{e}\|_{K^{\prime}}+\sum_{K^{\prime} \in \tilde{K}} \operatorname{osc}\left(\boldsymbol{f}, K^{\prime}\right)+\sum_{\gamma \in \tilde{\mathcal{F}}_{K} \cap \mathcal{F}_{N}} \operatorname{osc}(\boldsymbol{g}, \gamma)\right)
\end{aligned}
$$

where $\tilde{K}$ denotes the set of elements which share a vertex with element $K$ and $\tilde{\mathcal{F}}_{K}$ denotes the set of faces which share a vertex with element $K$.

Proof. The theorem is proved at the end of this section. 


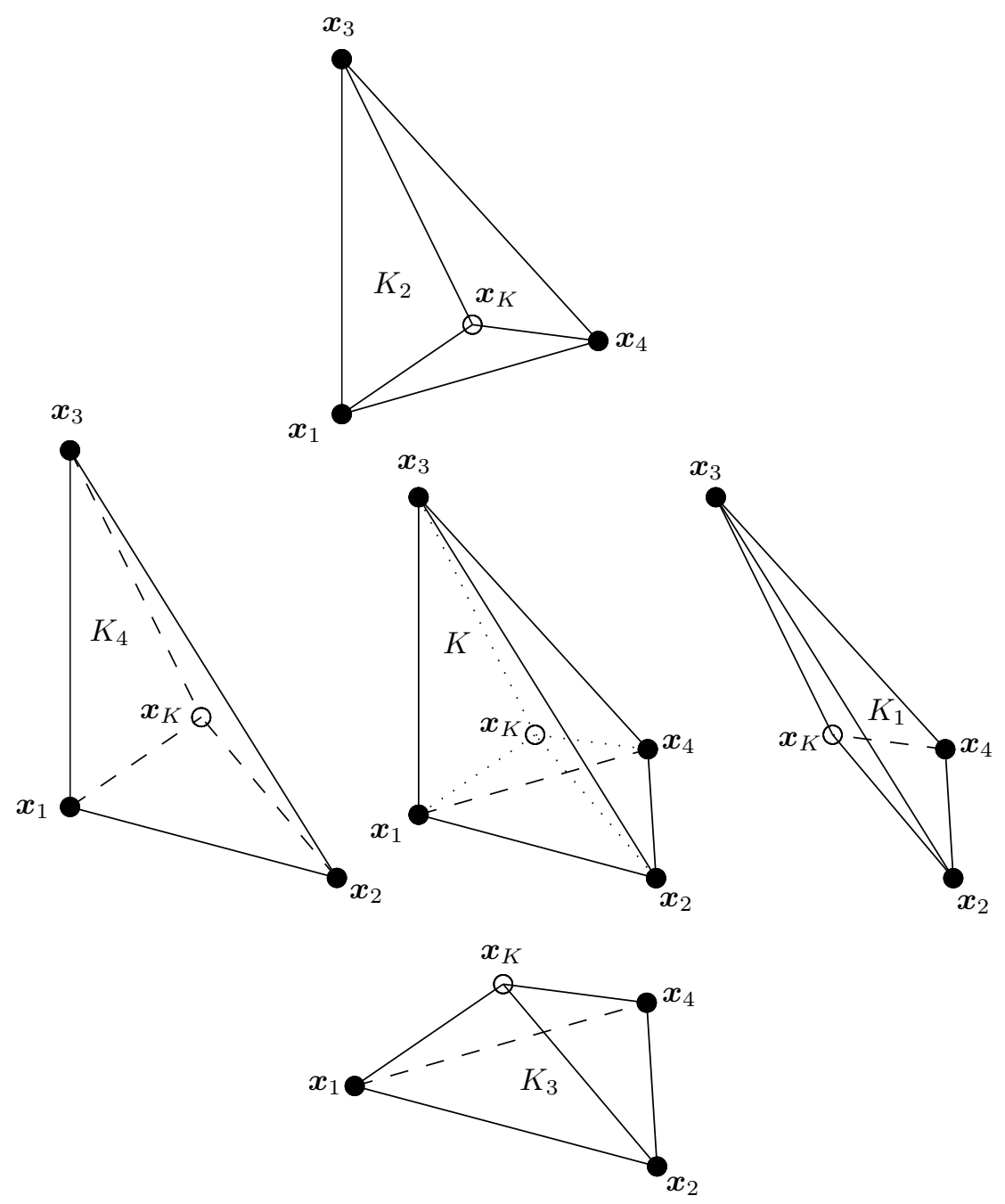

FIG. 3.2. The splitting of element $K$ (shown in Figure 3.1) into the four sub-tetrahedra $K_{1}, K_{2}, K_{3}$ and $K_{4}$ is shown in the centre, surrounded by individual diagrams of the four subtetrahedra. The centroid of the element $\boldsymbol{x}_{K}=\frac{1}{4}\left(\boldsymbol{x}_{1}+\boldsymbol{x}_{2}+\boldsymbol{x}_{3}+\boldsymbol{x}_{4}\right)$.

Theorem 3.5 asserts the existence of a field $\boldsymbol{\sigma}_{K} \in \underset{\boldsymbol{\sigma}_{K}}{\boldsymbol{V}}$ satisfying (3.32). For

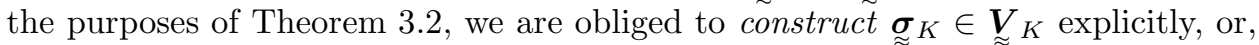
at any rate compute its norm. We construct $\boldsymbol{\sigma}_{K}$ on a given element $K$ in terms of functions $\underset{\approx}{\boldsymbol{\sigma}} \gamma$ associated with each face of $K$ as follows. Without loss of generality, we consider the face $\gamma_{4}$, with vertices $\boldsymbol{x}_{1}, \boldsymbol{x}_{2}$ and $\boldsymbol{x}_{3}$, of the element shown in Figure 3.1. For each vertex $\boldsymbol{x}_{1}, \boldsymbol{x}_{2}$ and $\boldsymbol{x}_{3}$ of the face we define three functions, which are associated with the remaining faces $\gamma_{1}, \gamma_{2}$ and $\gamma_{3}$. For example, in the case of vertex $\boldsymbol{x}_{1}$, the functions are denoted by $\underset{\sim}{\boldsymbol{\theta}_{\boldsymbol{n}_{1}, \boldsymbol{x}_{1}}^{\gamma_{4}},{\underset{\sim}{\boldsymbol{\theta}}}_{\boldsymbol{n}_{2}, \boldsymbol{x}_{1}}^{\gamma_{4}} \text { and } \underset{\sim}{\boldsymbol{\theta}_{3}, \boldsymbol{x}_{1}}}$ and satisfy the conditions:

$$
{\underset{\sim}{\boldsymbol{\theta}}}_{\boldsymbol{n}_{i}, \boldsymbol{x}_{1}}^{\gamma_{4}} \boldsymbol{n}_{\gamma^{\prime}}=\mathbf{0} \text { on } \gamma^{\prime} \in \mathcal{F}_{K} \backslash \gamma_{4}
$$

and

$$
\int_{\gamma_{4}}\left(\boldsymbol{n}_{\gamma^{\prime}}^{T} \underset{\sim}{\boldsymbol{\theta}_{n_{i}, \boldsymbol{x}_{1}}^{\gamma_{4}}} \boldsymbol{n}_{\gamma_{4}}\right) \lambda_{j} d \boldsymbol{S}= \begin{cases}\frac{1}{\left|\gamma_{i}\right|} & \text { if } j=1 \text { and } \gamma_{i}=\gamma^{\prime} \\ 0 & \text { otherwise }\end{cases}
$$


The function $\underset{\sim}{\boldsymbol{\theta}_{\boldsymbol{n}_{i}, \boldsymbol{x}_{1}}^{\gamma_{4}}}$ is assigned a weight $R_{\boldsymbol{n}_{i}, \boldsymbol{x}_{1}}^{\gamma_{4}}$, given in terms of the residual $\boldsymbol{R}_{\gamma_{4}, K}$ defined by (3.15), as follows:

$$
R_{\boldsymbol{n}_{i}, \boldsymbol{x}_{1}}^{\gamma_{4}}=\left|\gamma_{i}\right| \int_{\gamma_{4}}\left(\boldsymbol{n}_{\gamma_{i}}^{T} \boldsymbol{R}_{\gamma_{4}, K}\right) \lambda_{1} d \boldsymbol{S}
$$

The sum of this weighted combination yields the function

$$
\underset{\approx}{\boldsymbol{\theta}_{1}} \boldsymbol{x}_{\boldsymbol{x}_{1}}=\sum_{i=1}^{3} R_{\boldsymbol{n}_{i}, \boldsymbol{x}_{1}}^{\gamma_{4}} \underset{\boldsymbol{n}_{i}, \boldsymbol{x}_{1}}{\gamma_{4}}
$$

associated with vertex $\boldsymbol{x}_{1}$ of face $\gamma_{4}$. Functions associated with the vertices $\boldsymbol{x}_{2}$ and $\boldsymbol{x}_{3}$ of face $\gamma_{4}$ can be constructed similarly and used to construct a function associated with face $\gamma_{4}$ as follows:

$$
\underset{\approx}{\gamma_{K}^{\gamma_{4}}}=\sum_{i=1}^{3}{\underset{\approx}{\boldsymbol{\theta}_{i}}}_{\gamma_{4}}
$$

Our actual stress representer is then given by the sum of this function and the corresponding functions on the remaining faces:

$$
\underset{\approx}{\boldsymbol{g}}=\underset{\approx}{\boldsymbol{\sigma}_{K}^{\gamma_{1}}}+\underset{\approx}{\boldsymbol{\sigma}_{K}^{\gamma_{2}}}+\underset{\approx}{\boldsymbol{\sigma}} \gamma_{K}^{\gamma_{3}}+{\underset{\approx}{\boldsymbol{\sigma}}}_{K}^{\gamma_{4}}
$$

However, as seen in Theorem 3.2, in order to compute the upper bound on the error, we need only compute the value of the number $\int_{K} \mathbb{E}^{-1}{\underset{\approx}{\boldsymbol{\sigma}_{K}}}_{\boldsymbol{\sigma}_{\boldsymbol{\sigma}}} d \boldsymbol{x}$ and not the actual field $\underset{\widetilde{\sigma}}{\boldsymbol{\sigma}_{K}}$ itself.

All of the above arguments depend on whether or not one can find functions ${\underset{\sim}{\boldsymbol{\theta}}}_{\boldsymbol{n}_{i}, \boldsymbol{x}_{j}}^{\gamma}$ for all $i, j \in \mathcal{N}(\gamma)$ and $\gamma \in \mathcal{F}_{K}$ such that

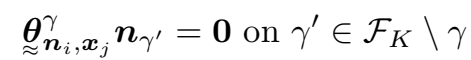

and, for $m \in \mathcal{N}(\gamma)$ and $\gamma^{\prime} \in \mathcal{F}_{K}$,

$$
\int_{\gamma}\left(\boldsymbol{n}_{\gamma^{\prime}}^{T} \underset{\approx}{\boldsymbol{\theta}_{\boldsymbol{n}_{i}, \boldsymbol{x}_{j}}^{\gamma}} \boldsymbol{n}_{\gamma}\right) \lambda_{m} d \boldsymbol{S}= \begin{cases}\frac{1}{\left|\gamma_{i}\right|} & \text { if } m=j \text { and } \gamma_{i}=\gamma^{\prime} \\ 0 & \text { otherwise. }\end{cases}
$$

In fact the following piecewise polynomials satisfy these properties:

The function $\underset{\sim}{\boldsymbol{\theta}_{\boldsymbol{n}_{1}, \boldsymbol{x}_{1}}^{\gamma_{4}}}$ is defined by

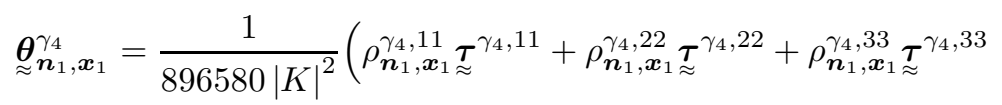

$$
\begin{aligned}
& \left.+\rho_{\boldsymbol{n}_{1}, \boldsymbol{x}_{1}}^{\gamma_{4}, 23} \underset{\approx}{\boldsymbol{\tau}^{\gamma_{4}, 23}}+\rho_{\boldsymbol{n}_{1}, \boldsymbol{x}_{1}}^{\gamma_{4}, 31} \underset{\approx}{\boldsymbol{\tau}^{\gamma_{4}, 31}}+\rho_{\boldsymbol{n}_{1}, \boldsymbol{x}_{1}}^{\gamma_{4}, 12} \boldsymbol{\tau}^{\gamma_{4}, 12}\right)
\end{aligned}
$$

where

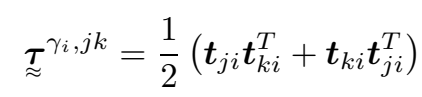

with

$$
\boldsymbol{t}_{j i}=\boldsymbol{x}_{j}-\boldsymbol{x}_{i}
$$

and the piecewise quadratic polynomials $\rho_{\boldsymbol{n}_{1}, \boldsymbol{x}_{1}}^{\gamma_{4}, 11}, \rho_{\boldsymbol{n}_{1}, \boldsymbol{x}_{1}}^{\gamma_{4}, 22}, \rho_{\boldsymbol{n}_{1}, \boldsymbol{x}_{1}}^{\gamma_{4}, 33}, \rho_{\boldsymbol{n}_{1}, \boldsymbol{x}_{1}}^{\gamma_{4}, 23}, \rho_{\boldsymbol{n}_{1}, \boldsymbol{x}_{1}}^{\gamma_{4}, 31}$ and

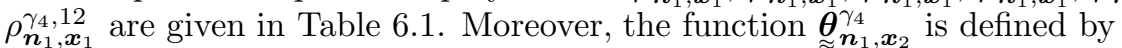

$$
\begin{aligned}
{\underset{\approx}{\boldsymbol{\theta}}}_{\boldsymbol{n}_{1}, \boldsymbol{x}_{2}}^{\gamma_{4}}=\frac{1}{36759780|K|^{2}} & \left(\rho_{\boldsymbol{n}_{1}, \boldsymbol{x}_{2}}^{\gamma_{4}, 11} \boldsymbol{\tau}^{\gamma_{4}, 11}+\rho_{\boldsymbol{n}_{1}, \boldsymbol{x}_{2}}^{\gamma_{4}, 22} \boldsymbol{\tau}^{\gamma_{4}, 22}+\rho_{\boldsymbol{n}_{1}, \boldsymbol{x}_{2}}^{\gamma_{4}, 33} \boldsymbol{\tau}^{\gamma_{4}, 33}\right. \\
& \left.+\rho_{\boldsymbol{n}_{1}, \boldsymbol{x}_{2}}^{\gamma_{4}, 23} \boldsymbol{\tau}^{\gamma_{4}, 23}+\rho_{\boldsymbol{n}_{1}, \boldsymbol{x}_{2}}^{\gamma_{4}, 31} \boldsymbol{\tau}^{\gamma_{4}, 31}+\rho_{\boldsymbol{n}_{1}, \boldsymbol{x}_{2}}^{\gamma_{4}, 12} \boldsymbol{\tau}^{\gamma_{4}, 12}\right)
\end{aligned}
$$


where the piecewise quadratic polynomials $\rho_{\boldsymbol{n}_{1}, \boldsymbol{x}_{2}}^{\gamma_{4}, 11}, \rho_{\boldsymbol{n}_{1}, \boldsymbol{x}_{2}}^{\gamma_{4}, 22}, \rho_{\boldsymbol{n}_{1}, \boldsymbol{x}_{2}}^{\gamma_{4}, 33}, \rho_{\boldsymbol{n}_{1}, \boldsymbol{x}_{2}}^{\gamma_{4}, 23}, \rho_{\boldsymbol{n}_{1}, \boldsymbol{x}_{2}}^{\gamma_{4}, 31}$ and $\rho_{\boldsymbol{n}_{1}, \boldsymbol{x}_{2}}^{\gamma_{4}, 12}$ are given in Table 6.2. In total, there are 36 distinct functions of the form ${\underset{\boldsymbol{\theta}}{\boldsymbol{\theta}, \boldsymbol{x}} \boldsymbol{\gamma}}_{\boldsymbol{\gamma}}$ corresponding to nine functions on each of the four faces of $K$, and the choices of $\boldsymbol{x}$ being any of the three vertices on the face itself, and $\boldsymbol{n}$ being the normals on the three remaining faces. Expressions for the remaining 34 functions can be obtained by permuting the indices of the functions $\underset{\sim}{\boldsymbol{\theta}_{\boldsymbol{n}_{1}, \boldsymbol{x}_{1}}^{\gamma_{4}}}$ and $\underset{\sim}{\boldsymbol{n}_{\boldsymbol{n}_{1}, \boldsymbol{x}_{2}}}$.

In general, for $\gamma \in \mathcal{F}_{K}$ and $i, j \in \mathcal{N}(\gamma)$,

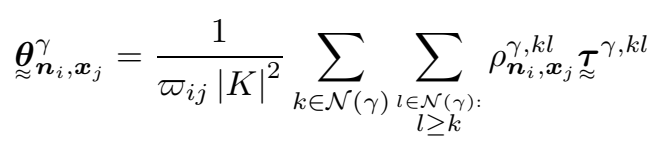

where

$$
\varpi_{i j}=\left\{\begin{aligned}
896580 & \text { if } i=j, \\
36759780 & \text { if } i \neq j,
\end{aligned}\right.
$$

and the piecewise quadratic polynomials $\rho_{\boldsymbol{n}_{i}, \boldsymbol{x}_{j}}^{\gamma, k l}$ can be obtained by permuting the indices of the $\rho_{\boldsymbol{n}_{1}, \boldsymbol{x}_{1}}^{\gamma, k l}$ given in Table 6.1 and the $\rho_{\boldsymbol{n}_{1}, \boldsymbol{x}_{2}}^{\gamma, k l}$ given in Table 6.2 for the cases $i=j$ and $i \neq j$ respectively. A tedious, but straightforward manipulation reveals that these functions do in fact satisfy (3.35) and (3.36). We note that there are other functions in $\underset{\widetilde{V}}{\boldsymbol{V}}$ which satisfy these properties. However, the choices given

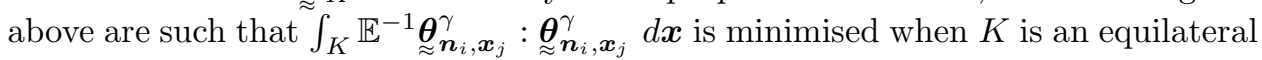
tetrahedron.

We are now in a position to give a proof of Theorem 3.5:

Proof. For $\gamma \in \mathcal{F}_{K}$, let

$$
\underset{\sim}{\underset{\sim}{\sigma}} \sum_{i \in \mathcal{N}(\gamma)} \sum_{j \in \mathcal{N}(\gamma)} \underset{\underset{\boldsymbol{n}_{i}, \boldsymbol{x}_{j}}{\gamma}}{\gamma} \int_{\gamma}\left|\gamma_{i}\right| \boldsymbol{n}_{\gamma_{i}}^{T} \boldsymbol{R}_{\gamma, K} \lambda_{j} d \boldsymbol{S} .
$$

Now, property (3.35) implies that $\boldsymbol{\sigma}_{K}^{\gamma} \boldsymbol{n}_{\gamma^{\prime}}$ vanishes on all faces $\gamma^{\prime}$ of element $K$ except face $\gamma$. Upon observing that $\left\{\lambda_{j}\right\}_{j \in \mathcal{N}(\gamma)}$ is a basis for $\mathbb{P}_{1}(\gamma)$ and that $\left\{\left|\gamma_{i}\right| \boldsymbol{n}_{\gamma_{i}}\right\}_{i \in \mathcal{N}(\gamma)}$ is a basis for $\mathbb{R}^{3}$, we see that $\left\{\left|\gamma_{i}\right| \boldsymbol{n}_{\gamma_{i}} \lambda_{j}\right\}_{i, j \in \mathcal{N}(\gamma)}$ is a basis for $\mathbb{P}_{1}(\gamma)^{3}$. Hence, it follows from property (3.36) that

$$
\int_{\gamma}\left({\underset{\approx}{\sigma}}_{K}^{\gamma} \boldsymbol{n}_{\gamma}\right) \cdot \boldsymbol{p} d \boldsymbol{S}=\int_{\gamma} \boldsymbol{R}_{\gamma, K} \cdot \boldsymbol{p} d \boldsymbol{S} \text { for all } \boldsymbol{p} \in \mathbb{P}_{1}(\gamma)^{3} .
$$

Hence, $\boldsymbol{\sigma}_{K}^{\gamma} \boldsymbol{n}_{\gamma}=\boldsymbol{R}_{\gamma, K}$ on face $\gamma$ of element $K$ since $\boldsymbol{R}_{\gamma, K} \in \mathbb{P}_{1}(\gamma)^{3}$ and the definition of $\underset{\approx}{\boldsymbol{V}} K$ means that $\underset{\approx}{\boldsymbol{\sigma}} \boldsymbol{n}_{\gamma} \in \mathbb{P}_{1}(\gamma)^{3}$. Hence,

$$
\underset{\sim}{\boldsymbol{g}} \boldsymbol{n}_{\gamma^{\prime}}^{\gamma}=\boldsymbol{R}_{\gamma, K} \delta_{\gamma \gamma^{\prime}} \text { on } \gamma^{\prime} \text { for all } \gamma^{\prime} \in \mathcal{F}_{K}
$$

from which (3.32) is a simple consequence when we construct $\boldsymbol{\sigma}_{K}$ as in (3.34).

Moreover, the triangle inequality allows us to say that

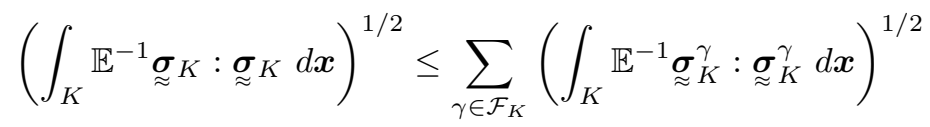

by the definition of $\underset{\sim}{\boldsymbol{\sigma}_{K}}(3.34)$ and

$$
\begin{aligned}
& \left(\int_{K} \mathbb{E}^{-1} \boldsymbol{\sigma}_{K}^{\gamma}: \boldsymbol{\sigma}_{K}^{\gamma} d \boldsymbol{x}\right)^{1 / 2} \\
& \leq \sum_{i \in \mathcal{N}(\gamma)} \sum_{j \in \mathcal{N}(\gamma)}\left|\int_{\gamma}\right| \gamma_{i}\left|\boldsymbol{n}_{\gamma_{i}}^{T} \boldsymbol{R}_{\gamma, K} \lambda_{j} d \boldsymbol{S}\right|\left(\int_{K} \mathbb{E}^{-1}{\underset{\sim}{\boldsymbol{\theta}}}_{\boldsymbol{n}_{i}, \boldsymbol{x}_{j}}^{\gamma}: \underset{\sim}{\boldsymbol{\theta}_{\boldsymbol{n}_{i}, \boldsymbol{x}_{j}}^{\gamma}} d \boldsymbol{x}\right)^{1 / 2}
\end{aligned}
$$


by the definition of $\underset{\approx}{\boldsymbol{\sigma}}{ }_{K}^{\gamma}(3.39)$. Now, standard arguments yield that

$$
\int_{K} \mathbb{E}^{-1}{\underset{\approx}{\boldsymbol{\theta}}}_{\boldsymbol{n}_{i}, \boldsymbol{x}_{j}}^{\gamma}:{\underset{\approx}{\boldsymbol{\theta}}}_{\boldsymbol{n}_{i}, \boldsymbol{x}_{j}}^{\gamma} d \boldsymbol{x} \leq C h_{K}^{-5}
$$

and

$$
\left|\int_{\gamma}\right| \gamma_{i}\left|\boldsymbol{n}_{\gamma_{i}}^{T} \boldsymbol{R}_{\gamma, K} \lambda_{j} d \boldsymbol{S}\right| \leq C h_{K}^{2}\left|\int_{\gamma} \boldsymbol{n}_{\gamma_{i}}^{T} \boldsymbol{R}_{\gamma, K} \lambda_{j} d \boldsymbol{S}\right| \leq C h_{K}^{3}\left\|\boldsymbol{R}_{\gamma, K}\right\|_{L_{2}(\gamma)} .
$$

Rewriting

$$
\boldsymbol{R}_{\gamma, K}=\boldsymbol{g}_{K, \gamma}-\left\langle\underset{\approx}{\boldsymbol{g}}\left(\boldsymbol{u}_{\boldsymbol{X}}\right) \boldsymbol{n}_{K}\right\rangle_{\gamma}-\boldsymbol{J}_{\gamma}
$$

where $\boldsymbol{J}_{\gamma}$ is defined by (3.12), allows us to say that

$$
\left\|\boldsymbol{R}_{\gamma, K}\right\|_{L_{2}(\gamma)} \leq\left\|\boldsymbol{g}_{K, \gamma}-\left\langle\underset{\approx}{\boldsymbol{g}}\left(\boldsymbol{u}_{\boldsymbol{X}}\right) \boldsymbol{n}_{K}\right\rangle_{\gamma}\right\|_{L_{2}(\gamma)}+\left\|\boldsymbol{J}_{\gamma}\right\|_{L_{2}(\gamma)}
$$

to which we can apply (3.9) and (3.11) to conclude that

$$
\begin{aligned}
& h_{K}^{3}\left\|\boldsymbol{R}_{\gamma, K}\right\|_{L_{2}(\gamma)} \\
\leq & C h_{K}^{5 / 2} \sum_{n \in \mathcal{N}(\gamma)}\left(\sum_{K^{\prime} \in \mathcal{P}_{n}}\|\boldsymbol{e}\|_{K^{\prime}}+\sum_{K^{\prime} \in \mathcal{P}_{n}} \operatorname{osc}\left(\boldsymbol{f}, K^{\prime}\right)+\sum_{\gamma^{\prime} \in \mathcal{F}_{n} \cap \mathcal{F}_{N}} \operatorname{osc}\left(\boldsymbol{g}, \gamma^{\prime}\right)\right) .
\end{aligned}
$$

Finally, by combining the above inequalities we can arrive at (3.33).

3.4. Bounding the oscillation terms. In order to bound the oscillation terms we shall require the three dimensional analog of the result proved for triangles in the appendix of [1].

Lemma 3.6. Let $w \in H^{1}(K)$ be such that $\int_{K} w d \boldsymbol{x}=0$. Then, for $\gamma \in \mathcal{F}_{K}$,

$$
\|w\|_{L_{2}(\gamma)} \leq\left(\frac{|\gamma|}{|K|} \frac{h_{K}}{\pi}\left(\frac{h_{K}}{\pi}+\frac{2}{3} \max _{\boldsymbol{x} \in \gamma}\left|\boldsymbol{x}-\boldsymbol{x}_{\gamma}\right|\right)\right)^{1 / 2}\|\operatorname{grad} w\|_{L_{2}(K)} .
$$

Proof. Let $\boldsymbol{\theta}_{\gamma}^{K}=\frac{|\gamma|}{3|K|}\left(\boldsymbol{x}-\boldsymbol{x}_{\gamma}\right)$ where $\boldsymbol{x}_{\gamma}$ is the vertex of element $K$ which does not lie on face $\gamma$. This function satisfies $\int_{\gamma^{\prime}} \boldsymbol{n}_{\gamma^{\prime}}^{K} \cdot \boldsymbol{\theta}_{\gamma}^{K} d \boldsymbol{S}=\delta_{\gamma \gamma^{\prime}}$ for all $\gamma^{\prime} \in \mathcal{F}_{K}$. Then

$$
\begin{aligned}
\|w\|_{L_{2}(\gamma)}^{2} & =\sum_{\gamma^{\prime} \in \mathcal{F}_{K}} \int_{\gamma^{\prime}} \boldsymbol{n}_{\gamma^{\prime}}^{K} \cdot \boldsymbol{\theta}_{\gamma}^{K} w^{2} d \boldsymbol{S}=\int_{K} \operatorname{div}\left(\boldsymbol{\theta}_{\gamma}^{K} w^{2}\right) d \boldsymbol{x} \\
& =\frac{|\gamma|}{|K|}\|w\|_{L_{2}(K)}^{2}+2 \int_{K} w \boldsymbol{\theta}_{\gamma}^{K} \cdot \operatorname{grad} w d \boldsymbol{x} \\
& \leq \frac{|\gamma|}{|K|}\|w\|_{L_{2}(K)}\left(\|w\|_{L_{2}(K)}+\frac{2}{3} \max _{\boldsymbol{x} \in \gamma}\left|\boldsymbol{x}-\boldsymbol{x}_{\gamma}\right|\|\operatorname{grad} w\|_{L_{2}(K)}\right) \\
& \leq \frac{|\gamma|}{|K|} \frac{h_{K}}{\pi}\left(\frac{h_{K}}{\pi}+\frac{2}{3} \max _{\boldsymbol{x} \in \gamma}\left|\boldsymbol{x}-\boldsymbol{x}_{\gamma}\right|\right)\|\operatorname{grad} w\|_{L_{2}(K)}^{2}
\end{aligned}
$$

upon applying the inequality

$$
\|w\|_{L_{2}(\gamma)} \leq \frac{h_{K}}{\pi}\|\operatorname{grad} w\|_{L_{2}(K)}
$$

which is proved in $[9,21]$.

We can then bound the oscillation terms as follows. 
Lemma 3.7. Let $C_{\gamma}^{K}$ be defined by (3.22). Then, for $\boldsymbol{v} \in \boldsymbol{H}_{D}^{1}(\Omega)$,

$$
\int_{K}\left(\boldsymbol{f}-P_{K} \boldsymbol{f}\right) \cdot \boldsymbol{v} d \boldsymbol{x} \leq \frac{1}{\pi} \operatorname{osc}(\boldsymbol{f}, K)\left(\int_{K} \underset{\approx}{\operatorname{grad}} \boldsymbol{v}: \underset{\approx}{\operatorname{grad}} \boldsymbol{v} d \boldsymbol{x}\right)^{1 / 2}
$$

for $K \in \mathcal{P}$ and

$$
\int_{\gamma}\left(\boldsymbol{g}-P_{\gamma} \boldsymbol{g}\right) \cdot \boldsymbol{v} d \boldsymbol{S} \leq C_{\gamma}^{K} \operatorname{osc}(\boldsymbol{g}, \gamma)\left(\int_{K} \underset{\approx}{\operatorname{grad} \boldsymbol{v}: \operatorname{grad} \boldsymbol{v} d \boldsymbol{x})^{1 / 2}}\right.
$$

for $\gamma \in \mathcal{F}_{K} \cap \mathcal{F}_{N}$.

Proof. Let $\boldsymbol{v}_{K} \in \mathbb{R}^{3}$ be such that

$$
\int_{K} \boldsymbol{p} \cdot\left(\boldsymbol{v}-\boldsymbol{v}_{K}\right) d \boldsymbol{x}=0 \text { for all } \boldsymbol{p} \in \mathbb{R}^{3} .
$$

Then, the definition of $P_{K} \boldsymbol{f}$ means that we can write

$$
\begin{aligned}
\int_{K}\left(\boldsymbol{f}-P_{K} \boldsymbol{f}\right) \cdot \boldsymbol{v} d \boldsymbol{x} & =\int_{K}\left(\boldsymbol{f}-P_{K} \boldsymbol{f}\right) \cdot\left(\boldsymbol{v}-\boldsymbol{v}_{K}\right) d \boldsymbol{x} \\
& \leq\left\|\boldsymbol{f}-P_{K} \boldsymbol{f}\right\|_{\boldsymbol{L}_{2}(K)}\left\|\boldsymbol{v}-\boldsymbol{v}_{K}\right\|_{\boldsymbol{L}_{2}(K)}
\end{aligned}
$$

from which (3.42) follows upon applying (3.41). Also, for $\gamma \in \mathcal{F}_{K} \cap \mathcal{F}_{N}$, the definition of $P_{\gamma} \boldsymbol{g}$ means that

$$
\begin{aligned}
\int_{\gamma}\left(\boldsymbol{g}-P_{\gamma} \boldsymbol{g}\right) \cdot \boldsymbol{v} d \boldsymbol{S} & =\int_{\gamma}\left(\boldsymbol{g}-P_{\gamma} \boldsymbol{g}\right) \cdot\left(\boldsymbol{v}-\boldsymbol{v}_{K}\right) d \boldsymbol{S} \\
& \leq\left\|\boldsymbol{g}-P_{\gamma} \boldsymbol{g}\right\|_{\boldsymbol{L}_{2}(\gamma)}\left\|\boldsymbol{v}-\boldsymbol{v}_{K}\right\|_{\boldsymbol{L}_{2}(\gamma)}
\end{aligned}
$$

to which we can apply (3.40) to arrive at (3.43).

We can also bound the oscillation terms in an alternative way.

Lemma 3.8. Let $C_{K}$ denote the constant in the local Korn's inequality (3.28). Then, for $\boldsymbol{v} \in \boldsymbol{H}_{D}^{1}(\Omega)$,

$$
\int_{K}\left(\boldsymbol{f}-P_{K} \boldsymbol{f}\right) \cdot \boldsymbol{v} d \boldsymbol{x} \leq \frac{C_{K}}{\pi} \operatorname{osc}(\boldsymbol{f}, K)\left(\int_{K} \underset{\approx}{\boldsymbol{\epsilon}}(\boldsymbol{v}): \underset{\sim}{\boldsymbol{\epsilon}}(\boldsymbol{v}) d \boldsymbol{x}\right)^{1 / 2}
$$

for $K \in \mathcal{P}$ and

$$
\int_{\gamma}\left(\boldsymbol{g}-P_{\gamma} \boldsymbol{g}\right) \cdot \boldsymbol{v} d \boldsymbol{S} \leq C_{K} C_{\gamma}^{K} \operatorname{osc}(\boldsymbol{g}, \gamma)\left(\int_{K} \underset{\approx}{\boldsymbol{\epsilon}}(\boldsymbol{v}): \underset{\approx}{\boldsymbol{\epsilon}}(\boldsymbol{v}) d \boldsymbol{x}\right)^{1 / 2}
$$

for $\gamma \in \mathcal{F}_{K} \cap \mathcal{F}_{N}$.

Proof. The definitions of $P_{K} \boldsymbol{f}$ and $P_{\gamma} \boldsymbol{g}$ mean that (3.42) and (3.43) will still hold with $\boldsymbol{v}$ on the right hand side replaced by $\boldsymbol{v}-\boldsymbol{v}_{K}$ where $\boldsymbol{v}_{K} \in \mathcal{R}(K)$. We can then apply (3.28) to the resulting inequalities to arrive at (3.44) and (3.45).

4. Practical application of the theory. Our main result is summarised in the following theorem.

ThEOREM 4.1. Let the error $\boldsymbol{e}=\boldsymbol{u}-\boldsymbol{u}_{\boldsymbol{X}}$ where $\boldsymbol{u}$ is the solution of (2.1) and $\boldsymbol{u}_{\boldsymbol{X}}$ is the solution of (2.3). Then

$$
\|\boldsymbol{e}\| \leq\left(\sum_{K \in \mathcal{P}} \int_{K} \mathbb{E}^{-1}{\underset{\sim}{\boldsymbol{g}}}_{K}:{\underset{\sim}{\boldsymbol{g}}}_{K} d \boldsymbol{x}\right)^{1 / 2}+\frac{C_{\Omega}}{\sqrt{2 \mu}}\left(\sum_{K \in \mathcal{P}} \Psi_{K}^{2}\right)^{1 / 2} .
$$


where $C_{\Omega}$ is the constant in (2.2) and $\Psi_{K}$ is defined by (3.21). Alternatively,

$$
\|\boldsymbol{e}\| \leq\left(\sum_{K \in \mathcal{P}}\left(\left(\int_{K} \mathbb{E}^{-1}{\underset{\sim}{\boldsymbol{\sigma}} K}_{K}:{\underset{\approx}{\boldsymbol{\sigma}}}_{K} d \boldsymbol{x}\right)^{1 / 2}+\frac{C_{K}}{\sqrt{2 \mu}} \Psi_{K}\right)^{2}\right)^{1 / 2}
$$

where $C_{K}$ is the constant in (3.28). Moreover, there exists a positive constant $C$, independent of the error $\boldsymbol{e}$ and the size of the elements in the mesh, such that

$$
\begin{aligned}
& \left(\int_{K} \mathbb{E}^{-1} \boldsymbol{\sigma}_{K}:{\underset{\sim}{\boldsymbol{\sigma}} K}_{K} d \boldsymbol{x}\right)^{1 / 2}+\frac{1}{\sqrt{2 \mu}} \max \left(C_{\Omega}, C_{K}\right) \Psi_{K} \\
\leq & C\left(\sum_{K^{\prime} \in \tilde{K}}\|\boldsymbol{e}\|_{K^{\prime}}+\sum_{K^{\prime} \in \tilde{K}} \operatorname{osc}\left(\boldsymbol{f}, K^{\prime}\right)+\sum_{\gamma \in \tilde{\mathcal{F}}_{K} \cap \mathcal{F}_{N}} \operatorname{osc}(\boldsymbol{g}, \gamma)\right)
\end{aligned}
$$

where $\tilde{K}$ denotes the set of elements which share a vertex with element $K$ and $\tilde{\mathcal{F}}_{K}$ denotes the set of faces which share a vertex with element $K$.

For the convenience of the reader who is primarily interested in the practicalities of implementing the estimator, we present an outline of the steps involved in the computation of $\int_{K} \mathbb{E}^{-1}{\underset{\sim}{\boldsymbol{\sigma}}}_{K}:{\underset{\sim}{\boldsymbol{\sigma}} K}_{K} d \boldsymbol{x}$.

The procedure for calculating $\int_{K} \mathbb{E}^{-1}{\underset{\sim}{\boldsymbol{g}_{K}}}_{\boldsymbol{\sigma}} \boldsymbol{\sigma}_{K} d \boldsymbol{x}$ is:

1. Calculate equilibrated stresses $\boldsymbol{g}_{K, \gamma}$ satisfying equations (3.2), (3.3), (3.4) and (3.9). A way of doing this is described in Section 3.1.

2. Use these equilibrated stresses to calculate the data defined by (3.15).

3. With these data, calculate $\int_{K} \mathbb{E}^{-1} \underset{\approx}{\boldsymbol{\sigma}_{K}}: \underset{\sim}{\boldsymbol{\sigma}_{K}} d \boldsymbol{x}$ (see below).

The final step is to calculate

$$
\begin{aligned}
& \int_{K} \mathbb{E}^{-1}{\underset{\sim}{\boldsymbol{\sigma}_{K}}}_{K}:{\underset{\sim}{\boldsymbol{\sigma}}}_{K} d \boldsymbol{x}=
\end{aligned}
$$

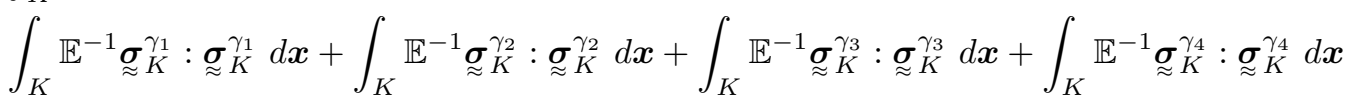

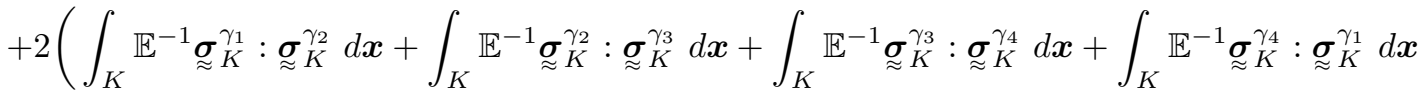

$$
\begin{aligned}
& \left.+\int_{K} \mathbb{E}^{-1} \underset{\approx}{\boldsymbol{\sigma}_{K}^{\gamma_{1}}}: \underset{\approx}{\boldsymbol{\sigma}_{K}^{\gamma_{3}}} d \boldsymbol{x}+\int_{K} \mathbb{E}^{-1}{\underset{\approx}{\boldsymbol{\sigma}}}_{K}^{\gamma_{2}}: \underset{\approx}{\boldsymbol{\sigma}_{K}^{\gamma_{4}}} d \boldsymbol{x}\right)
\end{aligned}
$$

The following lemma, which is proved in Section 6.1 of the appendix, shows how to compute this quantity without having to construct $\underset{\sim}{\boldsymbol{\sigma}_{K}}$ explicitly. We continue to use the notation shown in Figure 3.1. 
Lemma 4.2. Let $i, j, k, l \in\{1,2,3,4\}$ be distinct and define

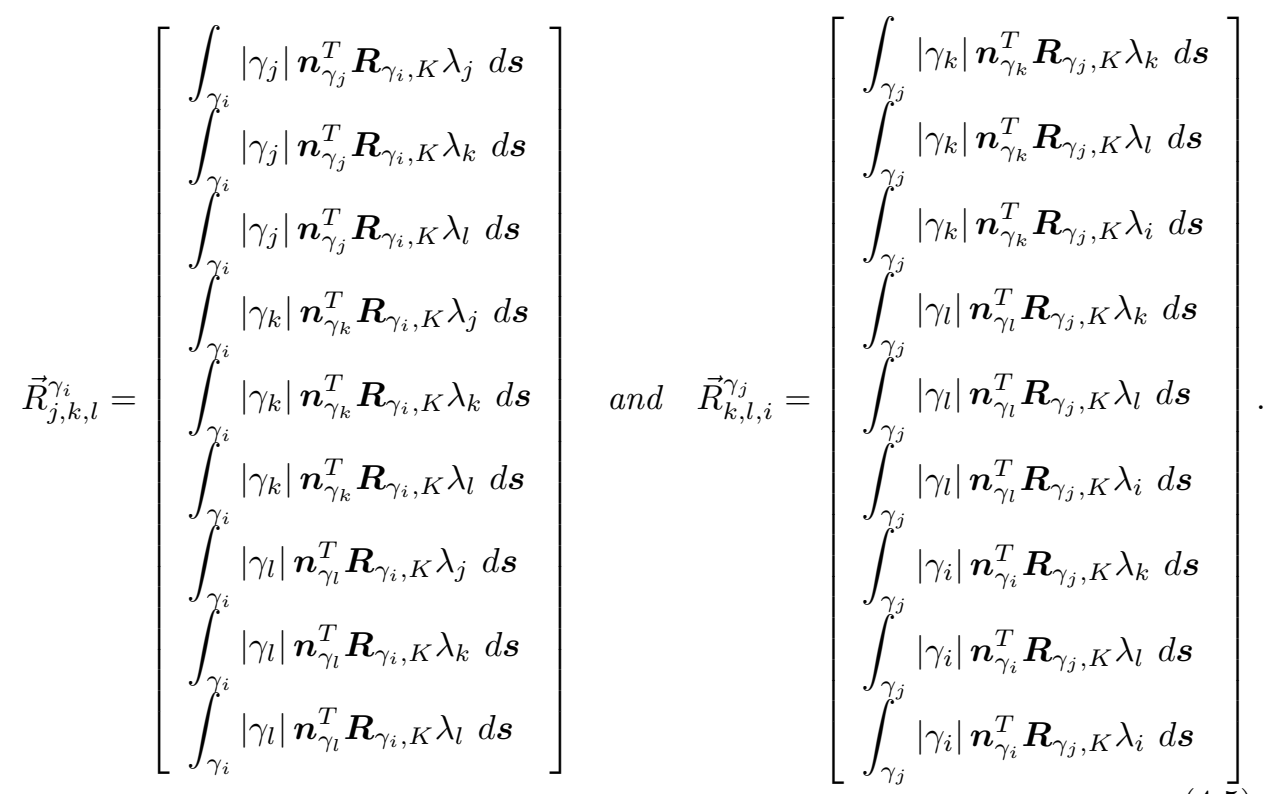

Then

$$
\int_{K} \mathbb{E}^{-1} \underset{\approx}{\boldsymbol{\sigma}_{K}^{\gamma_{i}}}: \underset{\approx}{\boldsymbol{\sigma}_{K}^{\gamma_{i}}} d \boldsymbol{x}=\frac{1}{|K|^{3}}\left(\vec{R}_{j, k, l}^{\gamma_{i}}\right)^{T} \boldsymbol{M}_{j, k, l}^{\gamma_{i}, \gamma_{i}} \vec{R}_{j, k, l}^{\gamma_{i}}
$$

and

$$
\int_{K} \mathbb{E}^{-1} \stackrel{\underset{\approx}{\boldsymbol{\sigma}} \gamma_{K}}{\gamma_{i}}:{\underset{\approx}{\boldsymbol{\sigma}_{K}}}_{K}^{\gamma_{j}} d \boldsymbol{x}=\frac{1}{|K|^{3}}\left(\vec{R}_{j, k, l}^{\gamma_{i}}\right)^{T} \boldsymbol{N}_{k, l}^{\gamma_{i}, \gamma_{j}} \vec{R}_{k, l, i}^{\gamma_{j}}
$$

where the matrix $\boldsymbol{M}_{j, k, l}^{\gamma_{i}, \gamma_{i}}$ is defined by

$$
\begin{aligned}
\boldsymbol{M}_{j, k, l}^{\gamma_{i}, \gamma_{i}}= & \boldsymbol{M}_{j j, j j} B_{j j, j j}^{\gamma_{i}}+\boldsymbol{M}_{j j, k k} B_{j j, k k}^{\gamma_{i}}+\boldsymbol{M}_{j j, l l} B_{j j, l l}^{\gamma_{i}}+\boldsymbol{M}_{j j, k l} B_{j j, k l}^{\gamma_{i}}+\boldsymbol{M}_{j j, l j} B_{j j, l j}^{\gamma_{i}} \\
& +\boldsymbol{M}_{j j, j k} B_{j j, j k}^{\gamma_{i}}+\boldsymbol{M}_{k k, k k} B_{k k, k k}^{\gamma_{i}}+\boldsymbol{M}_{k k, l l} B_{k k, l l}^{\gamma_{i}}+\boldsymbol{M}_{k k, k l} B_{k k, k l}^{\gamma_{i}}+\boldsymbol{M}_{k k, l j} B_{k k, l j}^{\gamma_{i}} \\
& +\boldsymbol{M}_{k k, j k} B_{k k, j k}^{\gamma_{i}}+\boldsymbol{M}_{l l, l l} B_{l l, l l}^{\gamma_{i}}+\boldsymbol{M}_{l l, k l} B_{l l, k l}^{\gamma_{i}}+\boldsymbol{M}_{l l, l j} B_{l l, l j}^{\gamma_{i}}+\boldsymbol{M}_{l l, j k} B_{l l, j k}^{\gamma_{i}} \\
& +\boldsymbol{M}_{k l, k l} B_{k l, k l}^{\gamma_{i}}+\boldsymbol{M}_{k l, l j} B_{k l, l j}^{\gamma_{i}}+\boldsymbol{M}_{k l, j k} B_{k l, j k}^{\gamma_{i}}+\boldsymbol{M}_{l j, l j} B_{l j, l j}^{\gamma_{i}}+\boldsymbol{M}_{l j, j k} B_{l j, j k}^{\gamma_{i}}+\boldsymbol{M}_{j k, j k} B_{j k, j k}^{\gamma_{i}}
\end{aligned}
$$

and the matrix $\boldsymbol{N}_{k, l}^{\gamma_{i}, \gamma_{j}}$ is defined by

$$
\begin{aligned}
\boldsymbol{N}_{k, l}^{\gamma_{i}, \gamma_{j}}= & \boldsymbol{N}_{j j, j j} B_{j j, j j}^{\gamma_{i}}+\boldsymbol{N}_{j j, k k} B_{j j, k k}^{\gamma_{i}}+\boldsymbol{N}_{j j, l l} B_{j j, l l}^{\gamma_{i}}+\boldsymbol{N}_{j j, k l} B_{j j, k l}^{\gamma_{i}}+\boldsymbol{N}_{j j, l j} B_{j j, l j}^{\gamma_{i}} \\
& +\boldsymbol{N}_{j j, j k} B_{j j, j k}^{\gamma_{i}}+\boldsymbol{N}_{k k, k k} B_{k k, k k}^{\gamma_{i}}+\boldsymbol{N}_{k k, l l} B_{k k, l l}^{\gamma_{i}}+\boldsymbol{N}_{k k, k l} B_{k k, k l}^{\gamma_{i}}+\boldsymbol{N}_{k k, l j} B_{k k, l j}^{\gamma_{i}} \\
& +\boldsymbol{N}_{k k, j k} B_{k k, j k}^{\gamma_{i}}+\boldsymbol{N}_{l l, l l} B_{l l, l l}^{\gamma_{i}}+\boldsymbol{N}_{l l, k l} B_{l l, k l}^{\gamma_{i}}+\boldsymbol{N}_{l l, l j} B_{l l, l j}^{\gamma_{i}}+\boldsymbol{N}_{l l, j k} B_{l l, j k}^{\gamma_{i}} \\
& +\boldsymbol{N}_{k l, k l} B_{k l, k l}^{\gamma_{i}}+\boldsymbol{N}_{k l, l j} B_{k l, l j}^{\gamma_{i}}+\boldsymbol{N}_{k l, j k} B_{k l, j k}^{\gamma_{i}}+\boldsymbol{N}_{l j, l j} B_{l j, l j}^{\gamma_{i}}+\boldsymbol{N}_{l j, j k} B_{l j, j k}^{\gamma_{i}}+\boldsymbol{N}_{j k, j k} B_{j k, j k}^{\gamma_{i}}
\end{aligned}
$$

where

$$
B_{o p, q r}^{\gamma_{i}}=\mathbb{E}^{-1} \underset{\approx}{\boldsymbol{\tau}_{i}, o p}:{\underset{\approx}{\boldsymbol{\tau}}}^{\gamma_{i}, q r}
$$

with

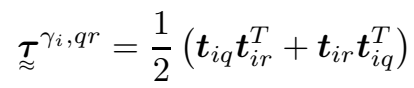

and

$$
\boldsymbol{t}_{i q}=\boldsymbol{x}_{q}-\boldsymbol{x}_{i}
$$


The matrices which appear in the definitions of $\boldsymbol{M}_{j, k, l}^{\gamma_{i}, \gamma_{i}}$ and $\boldsymbol{N}_{k, l}^{\gamma_{i}, \gamma_{j}}$ are defined in Tables 6.3 and 6.4 in the appendix.

By setting $i, j, k$ and $l$ to the appropriate values all ten terms on the right hand side of (4.4) can be calculated using the above lemma:

$$
\begin{aligned}
& i=1, j=2, k=3, l=4 \text { yields } \int_{K} \mathbb{E}^{-1} \underset{\approx}{\boldsymbol{\sigma}_{K}^{\gamma_{1}}}: \underset{\approx}{\boldsymbol{\sigma}_{K}^{\gamma_{1}}} d \boldsymbol{x} \text { and } \int_{K} \mathbb{E}^{-1}{\underset{\approx}{\boldsymbol{\sigma}_{K}}}_{K}^{\gamma_{1}}: \underset{\approx}{\boldsymbol{\sigma}} \gamma_{K}^{\gamma_{2}} d \boldsymbol{x} \\
& i=2, j=3, k=4, l=1 \text { yields } \int_{K} \mathbb{E}^{-1} \underset{\approx}{\boldsymbol{\sigma}} \gamma_{2}^{\gamma_{2}}: \underset{\approx}{\boldsymbol{\sigma}_{K}^{\gamma_{2}}} d \boldsymbol{x} \text { and } \int_{K} \mathbb{E}^{-1}{\underset{\approx}{\boldsymbol{\sigma}_{K}}}_{\gamma_{2}}^{\underset{\approx}{\boldsymbol{\sigma}} \gamma_{K}} d \boldsymbol{x} \\
& i=3, j=4, k=1, l=2 \text { yields } \int_{K} \mathbb{E}^{-1} \underset{\approx}{\underset{\approx}{\gamma_{3}}}: \underset{\approx}{\boldsymbol{\sigma}_{K}^{\gamma_{3}}} d \boldsymbol{x} \text { and } \int_{K} \mathbb{E}^{-1}{\underset{\approx}{\boldsymbol{\sigma}_{K}}}_{\gamma_{3}}^{\boldsymbol{\sigma}_{\approx}^{\gamma_{4}}} d \boldsymbol{x} \\
& i=4, j=1, k=2, l=3 \text { yields } \int_{K} \mathbb{E}^{-1} \underset{\approx}{\boldsymbol{\sigma}} \underset{K}{\gamma_{4}}: \underset{\approx}{\underset{\gamma_{4}}{\gamma_{4}}} d \boldsymbol{x} \text { and } \int_{K} \mathbb{E}^{-1}{\underset{\approx}{\boldsymbol{\sigma}^{\prime}}}_{\gamma_{4}}^{\boldsymbol{\sigma}_{\approx}^{\gamma_{1}}} d \boldsymbol{x} ; \\
& i=1, j=3, k=2, l=4 \text { yields } \int_{K} \mathbb{E}^{-1} \underset{\approx}{\boldsymbol{\sigma}_{K}}: \underset{\approx}{\boldsymbol{\sigma}_{K}^{\gamma_{3}}} d \boldsymbol{x} \\
& i=2, j=4, k=3, l=1 \text { yields } \int_{K} \mathbb{E}^{-1} \underset{\approx}{\boldsymbol{\sigma}_{K}^{\gamma_{2}}}: \underset{\approx}{\boldsymbol{\sigma}} \gamma_{K} d \boldsymbol{x} .
\end{aligned}
$$

5. Numerical example. Consider the problem $-\operatorname{div} \boldsymbol{\sigma}(\boldsymbol{u})=\mathbf{0}$ in the L-

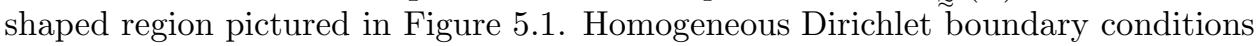
are imposed on the face which is built in. The Neumann boundary condition $\boldsymbol{g}=\left(\begin{array}{lll}0 & 0 & 1\end{array}\right)^{T}$ is imposed on the face on which there is a tangential traction. Homogeneous Neumann boundary conditions are imposed on the remaining faces. We took $\lambda=\frac{15}{26}$ and $\mu=\frac{5}{13}$. The initial mesh is shown in Figure 5.2(a).

For this example, both of the upper bounds given in Theorem 4.1 reduce to $\|\boldsymbol{e}\| \leq \eta$ where

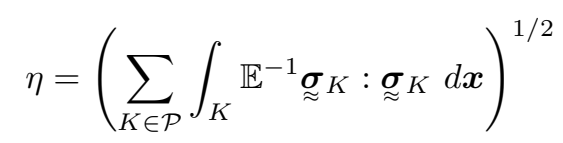

and $\int_{K} \mathbb{E}^{-1}{\underset{\approx}{\boldsymbol{\sigma}}}_{K}: \underset{\approx}{\boldsymbol{\sigma}_{K}} d \boldsymbol{x}$ is calculated using Lemma 4.2. However, as noted previously, the field $\underset{\approx}{\boldsymbol{\sigma}} \underset{K}{ }$ is not uniquely defined. We therefore also give results for when, on each element $K \in \mathcal{P}, \int_{K} \mathbb{E}^{-1}{\underset{\approx}{\boldsymbol{\sigma}}}_{K}:{\underset{\approx}{\boldsymbol{\sigma}}}_{K} d \boldsymbol{x}$ is minimised over $\underset{\approx}{\boldsymbol{V}} K$. We shall use $\eta_{\min }$ to denote the estimator obtained with this choice of ${\underset{\approx}{\sigma}}_{K}$.

We obtained results for a uniform refinement strategy as well as for an adaptive refinement strategy where we used a bulk criterion to refine the mesh on the smallest number of elements such that the sum of the contributions from these elements to the estimator of $\|\boldsymbol{e}\|^{2}$ exceeded $50 \%$ of the value of the estimator of $\|\boldsymbol{e}\|^{2}$. The final adaptively refined mesh is shown in Figure 5.2(b). We can see that the areas which have been the most refined are the re-entrant corner and the edges on which Dirichlet boundary conditions are imposed. The results obtained are shown in Figures 5.4, 5.5 and 5.6 where the effectivity indices $\vartheta=\eta /\|\boldsymbol{e}\|$ and $\vartheta_{\min }=\eta_{\min } /\|\boldsymbol{e}\|$.

In order to see how well our estimator performs we need to calculate the energy norm of the error. This proves problematic since the true solution to the problem that we are considering is unknown. However, we used the adaptive refinement strategy based on our estimator to refine the initial mesh 22 times to arrive at the mesh shown in Figure 5.3. The finite element approximation on this mesh gave us an approximate value of $\|\boldsymbol{u}\|^{2}$ which allowed us to calculate $\|\boldsymbol{e}\|^{2}=\|\boldsymbol{u}\|^{2}-\left\|\boldsymbol{u}_{\boldsymbol{X}}\right\|^{2}$ with sufficient accuracy for the results shown in Figures 5.4, 5.5 and 5.6 to give a faithful representation of the actual performance of the estimator.

In Figures 5.4(a), 5.5(a) and 5.6(a) it can be seen that the estimators do indeed provide guaranteed upper bounds on the energy norm of the error. It can also be seen that the error decreased at a faster rate when an adaptive refinement strategy 


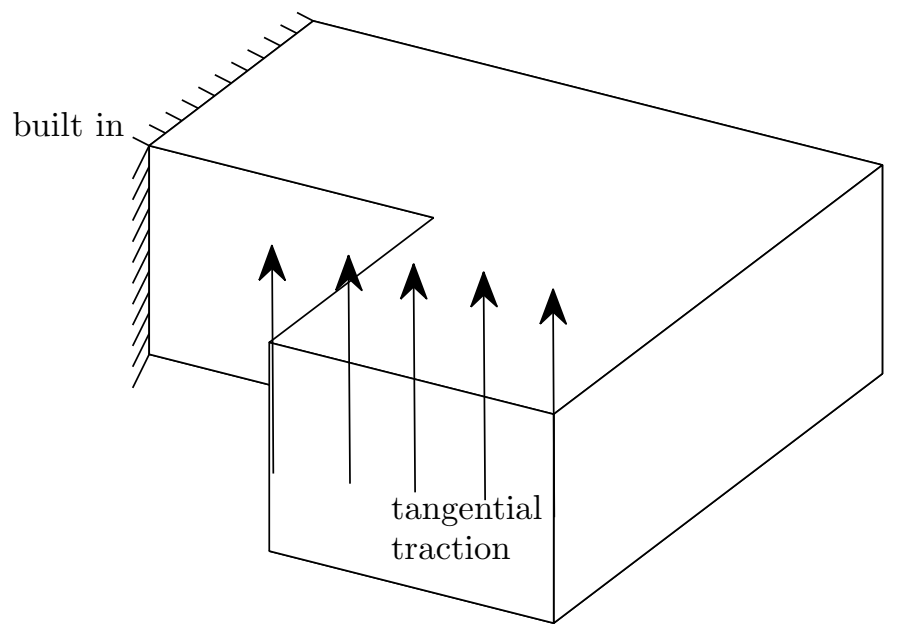

FIG. 5.1. Domain, loading and boundary conditions for the example.

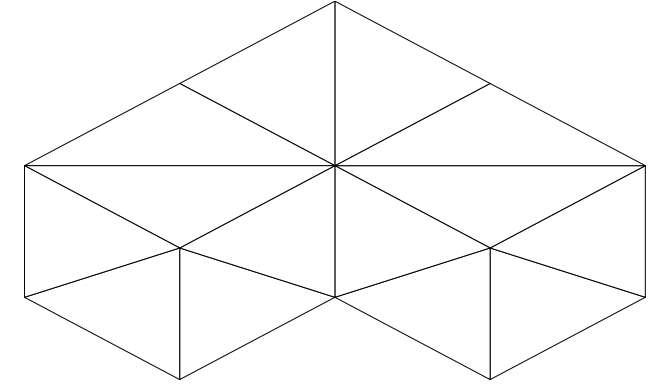

(a)

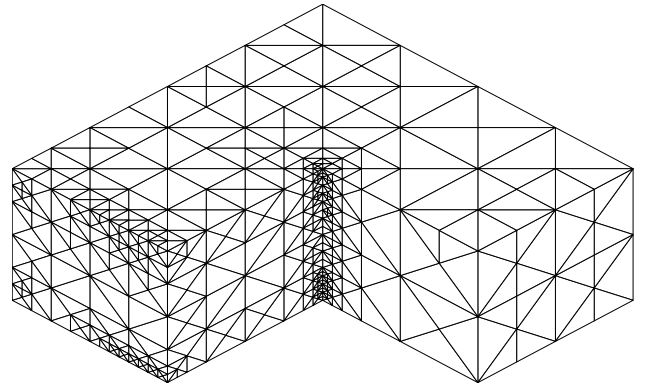

(b)

FIG. 5.2. The (a) initial and (b) final adaptively refined meshes for the example.

was used. Whether this strategy was based on the estimator $\eta$ or the estimator $\eta_{\text {min }}$ made negligible difference to the rate at which the error went down.

In Figures 5.4(b), 5.5(b) and 5.6(b) it can be seen that the estimators overestimated the energy norm of the error by a factor between 3.28 and 5.71 when uniform refinement was used. When adaptive refinement was used the energy norm of the error was overestimated by a factor between 3.74 and 4.55 by $\eta$ and a factor between 3.23 and 3.96 by $\eta_{\min }$. The difference in the amount of overestimation by the estimators $\eta$ and $\eta_{\min }$ varies between 0.31 and 0.97 . 


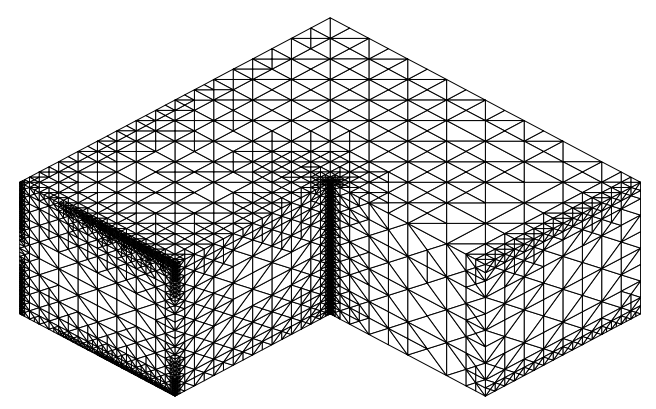

FIG. 5.3. The adaptively refined mesh containing 71377 tetrahedrons and 321426 degrees of freedom used to approximate $\|\boldsymbol{u}\|^{2}$.

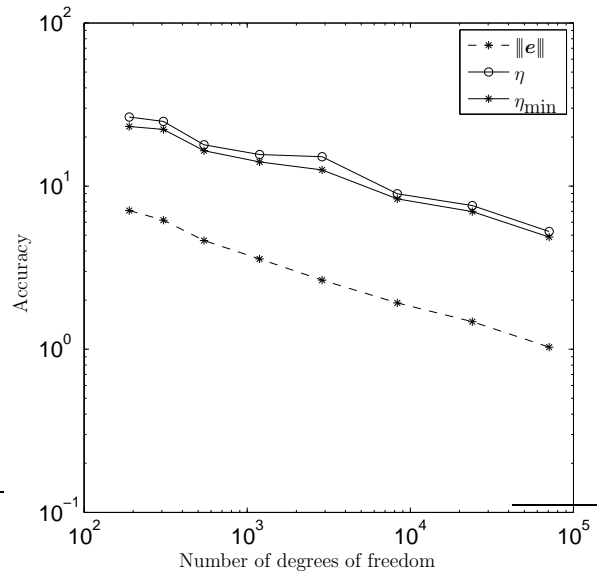

(a)

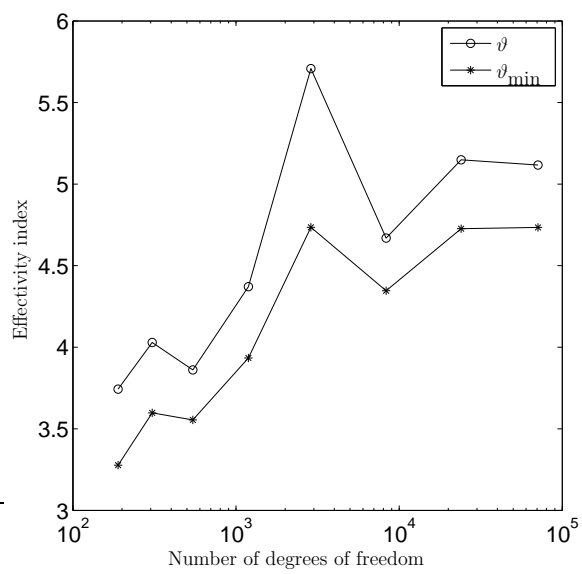

(b)

FIG. 5.4. The (a) performance and (b) effectivity indices of the estimators for the example with uniform refinement.

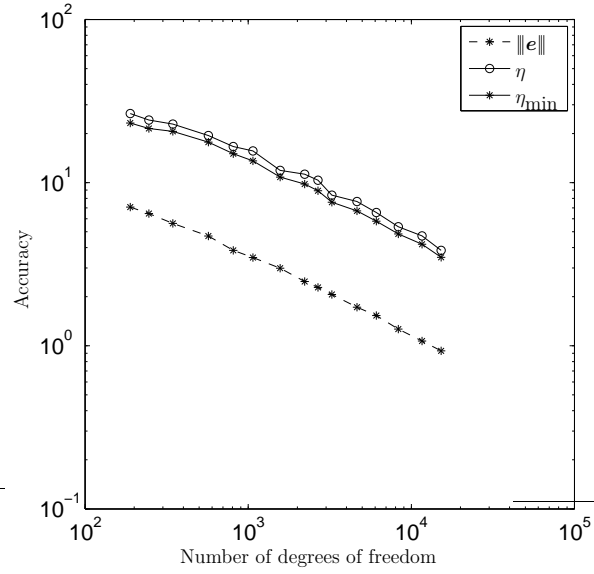

(a)

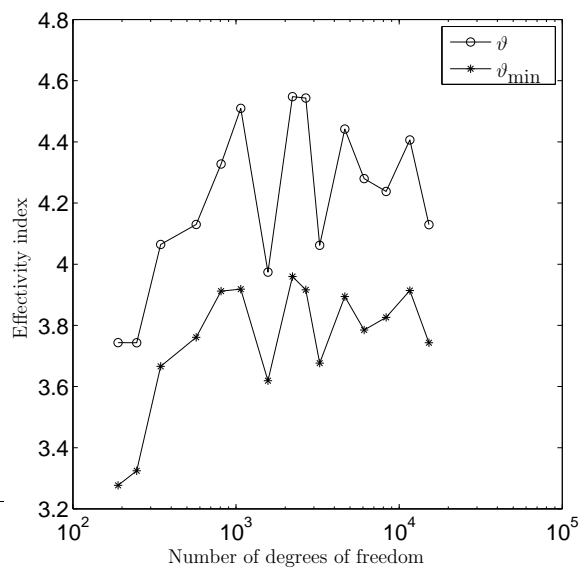

(b)

FIG. 5.5. The (a) performance and (b) effectivity indices of the estimators for the example with adaptive refinement with respect to $\eta$. 


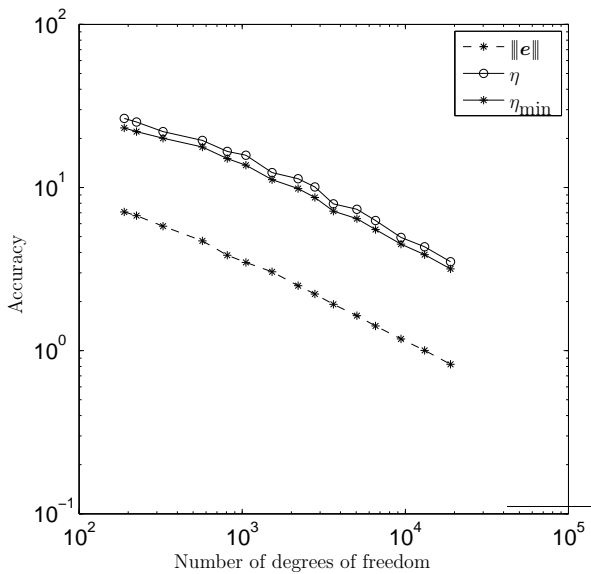

(a)

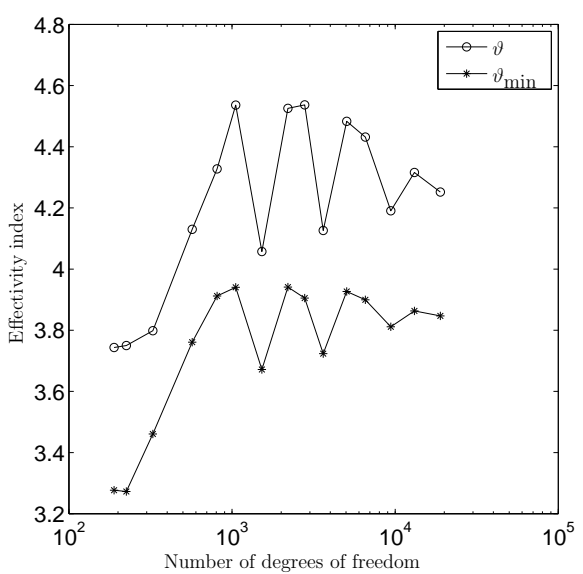

(b)

FIG. 5.6. The (a) performance and (b) effectivity indices of the estimators for the example with adaptive refinement with respect to $\eta_{\text {min }}$.

\section{Appendix.}

6.1. Proof of Lemma 4.2. Let the vertices, faces and unit normal vectors of element $K$ be as in Figure 3.1 and let $i, j, k, l \in\{1,2,3,4\}$ be distinct. Define

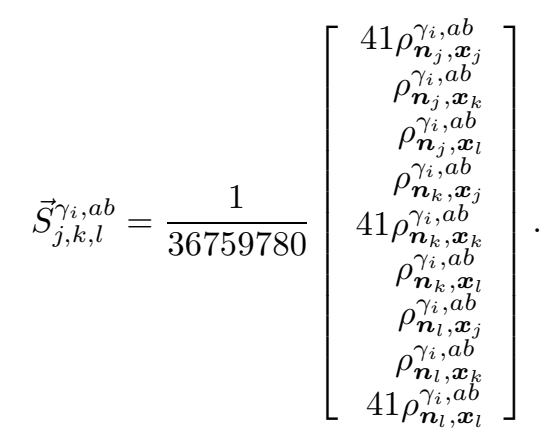

Then,

$$
\begin{aligned}
& \underset{\sim}{\boldsymbol{\sigma}_{K}^{\gamma_{i}}}=\frac{1}{|K|^{2}} \sum_{a \in \mathcal{N}\left(\gamma_{i}\right)} \sum_{\substack{b \in \mathcal{N}\left(\gamma_{i}\right): \\
b \geq a}}\left(\vec{R}_{j, k, l}^{\gamma_{i}}\right)^{T} \vec{S}_{j, k, l}^{\gamma_{i}, a b} \underset{\sim}{\boldsymbol{\tau}^{\gamma_{i}}, a b} \\
& =\frac{1}{|K|^{2}} \sum_{\substack { a \in \mathcal{N}\left(\gamma_{i}\right) \\
\begin{subarray}{c}{b \in \mathcal{N}\left(\gamma_{i}\right): \\
b \geq a{ a \in \mathcal { N } ( \gamma _ { i } ) \\
\begin{subarray} { c } { b \in \mathcal { N } ( \gamma _ { i } ) : \\
b \geq a } }\end{subarray}}\left(\vec{S}_{j, k, l}^{\gamma_{i}, a b}\right)^{T} \vec{R}_{j, k, l}^{\gamma_{i}} \overbrace{}^{\gamma_{i}, a b}
\end{aligned}
$$

Therefore,

$$
\int_{K} \mathbb{E}^{-1} \underset{\sim}{\boldsymbol{\sigma}_{K}}: \underset{\sim}{\gamma_{K}^{\gamma_{i}}} d \boldsymbol{x}=\frac{1}{|K|^{3}}\left(\vec{R}_{j, k, l}^{\gamma_{i}}\right)^{T} \boldsymbol{M}_{j, k, l}^{\gamma_{i}, \gamma_{i}} \vec{R}_{j, k, l}^{\gamma_{i}}
$$

where the matrix $\boldsymbol{M}_{j, k, l}^{\gamma_{i}, \gamma_{i}}$ is defined by

$$
\boldsymbol{M}_{j, k, l}^{\gamma_{i}, \gamma_{i}}=\sum_{a \in \mathcal{N}\left(\gamma_{i}\right)} \sum_{\substack{b \in \mathcal{N}\left(\gamma_{i}\right): c \in \mathcal{N}\left(\gamma_{i}\right) \\ b \geq a}} \sum_{\substack{d \in \mathcal{N}\left(\gamma_{i}\right): \\ d \geq c}} \frac{1}{|K|} \int_{K} \vec{S}_{j, k, l}^{\gamma_{i}, a b}\left(\vec{S}_{j, k, l}^{\gamma_{i}, c d}\right)^{T} d \boldsymbol{x} B_{a b, c d}^{\gamma_{i}}
$$


and

$$
\int_{K} \mathbb{E}^{-1} \underset{\approx}{\boldsymbol{\sigma}_{K}^{\gamma_{i}}}: \underset{\sim}{\boldsymbol{\sigma}_{K}^{\gamma_{j}}} d \boldsymbol{x}=\frac{1}{|K|^{3}}\left(\vec{R}_{j, k, l}^{\gamma_{i}}\right)^{T} \boldsymbol{N}_{k, l}^{\gamma_{i}, \gamma_{j}} \vec{R}_{k, l, i}^{\gamma_{j}}
$$

where the matrix $\boldsymbol{N}_{k, l}^{\gamma_{i}, \gamma_{j}}$ is defined by

$$
\boldsymbol{N}_{k, l}^{\gamma_{i}, \gamma_{j}}=\sum_{a \in \mathcal{N}\left(\gamma_{i}\right)} \sum_{\substack{b \in \mathcal{N}\left(\gamma_{i}\right): c \in \mathcal{N}\left(\gamma_{j}\right) \\ b \geq a}} \sum_{\substack{d \in \mathcal{N}\left(\gamma_{j}\right): \\ d \geq c}} \frac{1}{|K|} \int_{K} \vec{S}_{j, k, l}^{\gamma_{i}, a b}\left(\vec{S}_{k, l, i}^{\gamma_{j}, c d}\right)^{T} d \boldsymbol{x}\left(\mathbb{E}^{-1}{\underset{\sim}{\boldsymbol{\tau}}}^{\gamma_{i}, a b}: \underset{\sim}{\boldsymbol{\tau}^{\gamma_{j}, c d}}\right) .
$$

Now, since $i, j, k$ and $l$ are distinct and $a, b, c, d \in\{i, j, k, l\}$, for fixed $a, b, c$ and $d$ the entries in the $9 \times 9$ matrices

$$
\frac{1}{|K|} \int_{K} \vec{S}_{j, k, l}^{\gamma_{i}, a b}\left(\vec{S}_{j, k, l}^{\gamma_{i}, c d}\right)^{T} d \boldsymbol{x}
$$

and

$$
\frac{1}{|K|} \int_{K} \vec{S}_{j, k, l}^{\gamma_{i}, a b}\left(\vec{S}_{k, l, i}^{\gamma_{j}, c d}\right)^{T} d \boldsymbol{x}
$$

will be independent of the particular values taken by $i, j, k$ and $l$. These matrices are also independent of which element $K$ we are on. Hence, these matrices need only be calculated once for each admissible combination of values for $a, b, c$ and $d$.

Hence, upon also making use of the property that

$$
B_{a b, c d}^{\gamma_{i}}=B_{b a, c d}^{\gamma_{i}}=B_{a b, d c}^{\gamma_{i}}=B_{c d, a b}^{\gamma_{i}}
$$

we can conclude that (4.6) holds with $\boldsymbol{M}_{j, k, l}^{\gamma_{i}, \gamma_{i}}$ defined by (4.8). Similarly, upon also making use of the fact that $\boldsymbol{t}_{j k}=\boldsymbol{t}_{i k}-\boldsymbol{t}_{i j}, \boldsymbol{t}_{j l}=\boldsymbol{t}_{i l}-\boldsymbol{t}_{i j}$ and $\boldsymbol{t}_{j i}=-\boldsymbol{t}_{i j}$ to rewrite the $\left(\mathbb{E}^{-1}{\underset{\sim}{\tau_{i}}}^{\gamma_{i}, a b}:{\underset{\sim}{\boldsymbol{\tau}}}^{\gamma_{j}, c d}\right)$ in terms of $B_{a b, c d}^{\gamma_{i}}$, we can conclude that (4.7) holds with $\boldsymbol{N}_{k, l}^{\gamma_{i}, \gamma_{j}}$ defined by $(4.9)$.

7. Acknowledgements. The authors wish to express their gratitude to Prof. C. Horgan for his correspondence regarding Korn's inequality. The authors also wish to thank Mr. R. Campbell of Ideas Ltd for a number of stimulating discussions on the issue of quality control and accuracy assessment in finite element analysis. We are indebted to Dr. X. Ma for providing us with his three dimensional mesh refinement software.

\section{REFERENCES}

[1] M. Ainsworth. A posteriori error estimation for discontinuous Galerkin finite element approximation. SIAM J. Numer. Anal., 45(4):1777-1798 (electronic), 2007.

[2] M. Ainsworth, L. Demkowicz, and C.-W. Kim. Analysis of the equilibrated residual method for a posteriori error estimation on meshes with hanging nodes. Comput. Methods Appl. Mech. Engrg., 196(37-40):3493-3507, 2007.

[3] M. Ainsworth and J. T. Oden. A Posteriori Error Estimation in Finite Element Analysis. Pure and Applied Mathematics. Wiley-Interscience, John Wiley \& Sons, New York, 2000.

[4] M. Ainsworth and R. Rankin. Guaranteed computable error bounds for conforming and nonconforming finite element analyses in planar elasticity. Internat. J. Numer. Methods Engrg., to appear.

[5] D. N. Arnold, J. Douglas, Jr., and C. P. Gupta. A family of higher order mixed finite element methods for plane elasticity. Numer. Math., 45(1):1-22, 1984.

[6] D. N. Arnold and R. Winther. Mixed finite elements for elasticity. Numer. Math., 92(3):401419, 2002.

[7] I. Babuška and A. Miller. A feedback finite element method with a posteriori error estimation. I. The finite element method and some basic properties of the a posteriori error estimator. Comput. Methods Appl. Mech. Engrg., 61(1):1-40, 1987. 


\begin{tabular}{|c|c|c|c|c|c|c|c|c|c|c|c|}
\hline & $\lambda_{1}^{2}$ & $\lambda_{2}^{2}$ & $\lambda_{3}^{2}$ & $\overline{\lambda_{4}^{2}}$ & $\lambda_{1} \lambda_{2}$ & $\lambda_{2} \lambda_{3}$ & $\lambda_{3} \lambda_{4}$ & $\lambda_{4} \lambda_{1}$ & $\lambda_{1} \lambda_{3}$ & $\lambda_{2} \lambda_{4}$ & \\
\hline$\rho_{\boldsymbol{n}_{1}, \boldsymbol{x}_{1}}^{\gamma_{4}, 11}$ & -4590 & 0 & 0 & 0 & 1195440 & 0 & 0 & 1195440 & 1195440 & 0 & \\
\hline $\begin{array}{l}\rho_{\boldsymbol{n}_{1}}^{\gamma_{4}, \boldsymbol{x}_{1}} \\
\boldsymbol{\gamma}_{1}\end{array}$ & 821364 & 896580 & 0 & 0 & -1562836 & 115846 & 0 & 261306 & -239793 & -205937 & \\
\hline$\rho_{\boldsymbol{n}_{1}, \boldsymbol{x}_{1}}^{\gamma_{4}, \boldsymbol{x}_{1}}$ & 821364 & 0 & 896580 & 0 & -239793 & 115846 & -205937 & 261306 & -1562836 & 0 & on \\
\hline$\rho_{\boldsymbol{n}_{1}, \boldsymbol{x}_{1}}^{\gamma_{4}, \omega_{1}}$ & 2695160 & -298860 & -298860 & 0 & -754756 & 963748 & 898338 & -3204972 & -754756 & 898338 & $K_{1}$ \\
\hline$\rho_{\boldsymbol{n}_{1}, \boldsymbol{x}_{1}}^{\gamma_{4}, w_{1}}$ & 355802 & 0 & 0 & 0 & 1264366 & 0 & 0 & -486464 & -4063604 & 0 & \\
\hline $\begin{array}{l}\rho_{n_{1},}^{n_{1}, 12} \\
\rho_{\boldsymbol{n}_{1}, \boldsymbol{x}_{1}}\end{array}$ & 355802 & 0 & 0 & 0 & -4063604 & 0 & 0 & -486464 & 1264366 & 0 & \\
\hline$\rho_{\boldsymbol{n}_{1}, \boldsymbol{x}_{1}}^{\gamma_{4}, 11}$ & -896580 & -1805544 & 0 & 0 & 3824396 & 1628106 & 0 & 1943210 & -308719 & -803139 & \\
\hline$\rho_{\boldsymbol{n}_{1}, \boldsymbol{x}_{1}}^{\gamma_{4}, \boldsymbol{x}_{1}}$ & 0 & 86530 & 0 & 0 & 0 & 0 & 0 & 0 & 0 & 0 & \\
\hline$\rho_{\boldsymbol{n}_{1}, \boldsymbol{x}_{1}}^{\gamma_{4}, w_{1}}$ & 0 & -118950 & 0 & 0 & 785137 & 205480 & 759517 & 0 & -785137 & -759517 & on \\
\hline$\rho_{\boldsymbol{n}_{1}, \boldsymbol{x}_{1}}^{\gamma_{4}, \boldsymbol{x}_{1}}$ & 0 & -32420 & 0 & 0 & 1570274 & 124560 & 0 & 0 & 0 & -1519034 & $K_{2}$ \\
\hline$\rho_{\boldsymbol{n}_{1}, \boldsymbol{x}_{1}}^{\gamma_{4}, w_{1}}$ & 0 & 4599530 & 298860 & 0 & -3083052 & -4644172 & -898338 & 0 & 496136 & 301136 & \\
\hline$\rho_{\boldsymbol{n}_{1}, \boldsymbol{x}_{1}}^{\gamma_{4}, \omega_{12}}$ & 0 & -1258042 & 0 & 0 & -2586916 & 1512260 & 0 & 0 & 0 & -597202 & \\
\hline$\rho_{\boldsymbol{n}_{1}, \boldsymbol{x}_{1}}^{\gamma_{4}, 11}$ & -896580 & 0 & -1805544 & 0 & -308719 & 1628106 & -803139 & 1943210 & 3824396 & 0 & \\
\hline$\rho_{\boldsymbol{n}_{1}, \boldsymbol{x}_{1}}^{\gamma_{4}, x_{1}}$ & 0 & 0 & -118950 & 0 & -785137 & 205480 & -759517 & 0 & 785137 & 759517 & \\
\hline$\rho_{\boldsymbol{n}_{1}, \boldsymbol{x}_{1}}^{\gamma_{4}, x_{1}}$ & 0 & 0 & 86530 & 0 & 0 & 0 & 0 & 0 & 0 & 0 & on \\
\hline$\rho_{\boldsymbol{n}_{1}, \boldsymbol{x}_{1}}^{\gamma_{4}, \boldsymbol{x}_{1}}$ & 0 & 0 & -32420 & 0 & 0 & 124560 & -1519034 & 0 & 1570274 & 0 & $K_{3}$ \\
\hline$\rho_{\boldsymbol{n}_{1}, \boldsymbol{x}_{1}}^{\gamma_{4}, w_{1}}$ & 0 & 0 & -1258042 & 0 & 0 & 1512260 & -597202 & 0 & -2586916 & 0 & \\
\hline$\rho_{\boldsymbol{n}_{1}, \boldsymbol{x}_{1}}^{\gamma_{4}, 1_{1}}$ & 0 & 298860 & 4599530 & 0 & 496136 & -4644172 & 301136 & 0 & -3083052 & -898338 & \\
\hline$\rho_{\boldsymbol{n}_{1}, \boldsymbol{x}_{1}}^{\gamma_{4}, 11}$ & -896580 & -298860 & -298860 & -6934182 & -1580941 & -597720 & 3854283 & 8061248 & -1580941 & 3854283 & \\
\hline$\rho_{\boldsymbol{n}_{1}, \boldsymbol{x}_{1}}^{\gamma_{4}, x_{1}}$ & 0 & -896580 & 0 & -1067064 & 90917 & -134666 & 251813 & -153086 & 0 & 1995196 & \\
\hline$\rho_{\boldsymbol{n}_{1}, \boldsymbol{x}_{1}}^{\gamma_{4}, x_{1}}$ & 0 & 0 & -896580 & -1067064 & 0 & -134666 & 1995196 & -153086 & 90917 & 251813 & on \\
\hline$\rho_{\boldsymbol{n}_{1}, \boldsymbol{x}_{1}}^{\gamma_{4}, 23}$ & 0 & 298860 & 298860 & -3339840 & -1074138 & -926108 & 1182836 & 3594212 & -1074138 & 1182836 & $K_{4}$ \\
\hline$\rho_{\boldsymbol{n}_{1}, \boldsymbol{x}_{1}}^{\gamma_{4}, w_{1}}$ & 0 & -298860 & 1494300 & 5970410 & 1074138 & 1195440 & -8793352 & -4180344 & 892304 & -283936 & \\
\hline$\rho_{\boldsymbol{n}_{1}, \boldsymbol{x}_{1}}^{\gamma_{4}, \boldsymbol{x}_{1}}$ & 0 & 1494300 & -298860 & 5970410 & 892304 & 1195440 & -283936 & -4180344 & 1074138 & -8793352 & \\
\hline
\end{tabular}

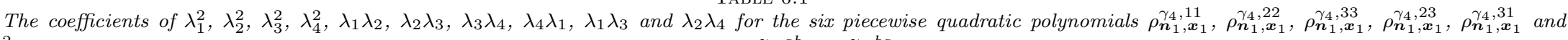
$\rho_{\boldsymbol{n}_{1}, \boldsymbol{x}_{1}}^{\gamma_{4}, 12}$ in (3.37) on each of the four sub-tetrahedra $K_{1}, K_{2}, K_{3}$ and $K_{4}$. We define $\rho_{\boldsymbol{n}_{1}, \boldsymbol{x}_{1}}^{\gamma_{4}, a b}=\rho_{\boldsymbol{n}_{1}, \boldsymbol{x}_{1}}^{\gamma_{4}, b a}$. 


\begin{tabular}{|c|c|c|c|c|c|c|c|c|c|c|c|}
\hline & $\lambda_{1}^{2}$ & $\lambda_{2}^{2}$ & $\lambda_{3}^{2}$ & $\lambda_{4}^{2}$ & $\lambda_{1} \lambda_{2}$ & $\lambda_{2} \lambda_{3}$ & $\lambda_{3} \lambda_{4}$ & $\lambda_{4} \lambda_{1}$ & $\lambda_{1} \lambda_{3}$ & $\lambda_{2} \lambda_{4}$ & \\
\hline$\rho_{\boldsymbol{n}_{1}, \boldsymbol{x}_{2}}^{\gamma_{4}, 11}$ & -15612800 & 0 & 0 & 0 & -196052160 & 0 & 0 & 49013040 & 49013040 & 0 & \\
\hline$\rho_{\boldsymbol{n}_{1}, \boldsymbol{x}_{2}}^{\gamma_{4},{ }^{2}}$ & -97196160 & -110279340 & 0 & 0 & 220871840 & 2772867 & 0 & -15802163 & -2447827 & 2080783 & \\
\hline$\rho_{\boldsymbol{n}_{1}, \boldsymbol{x}_{2}}^{\gamma_{4}, \boldsymbol{x}_{2}}$ & 19770612 & 0 & 36759780 & 0 & 22603673 & -13043859 & 10090231 & -1187909 & -74992528 & 0 & on \\
\hline$\rho_{\boldsymbol{n}_{1}, \boldsymbol{x}_{2}}^{\gamma_{4}, \boldsymbol{x}_{2}}$ & -123717500 & 36759780 & -12253260 & 0 & 26663970 & -38742048 & 10971678 & 139383272 & 52777302 & -91843194 & $K_{1}$ \\
\hline$\rho_{\boldsymbol{n}_{1}, \boldsymbol{x}_{2}}^{\gamma_{4}, x_{1}}$ & 165995636 & 0 & 0 & 0 & -139275036 & 0 & 0 & 71662732 & -77144932 & 0 & \\
\hline$\rho_{\boldsymbol{n}_{1}, \boldsymbol{x}_{2}}^{\gamma_{4}, 12}$ & -200937640 & 0 & 0 & 0 & 392177780 & 0 & 0 & -15133244 & 29932464 & 0 & \\
\hline $\begin{array}{l}\rho_{\boldsymbol{n}_{1}, \boldsymbol{x}_{2}}^{\gamma_{4}, 11} \\
\boldsymbol{\gamma}_{1}\end{array}$ & 12253260 & -68507640 & 0 & 0 & -168806920 & 32055251 & 0 & 48344121 & 16632749 & 14390299 & \\
\hline$\rho_{\boldsymbol{n}_{1}, \boldsymbol{x}_{2}}^{\gamma_{4}, \boldsymbol{x}^{2}}$ & 0 & 0 & 0 & 0 & 0 & 0 & 0 & 0 & 0 & 0 & \\
\hline$\rho_{\boldsymbol{n}_{1}, \boldsymbol{x}_{2}}^{\gamma_{4}, \boldsymbol{x}_{2}}$ & 0 & 0 & 0 & 0 & 47685439 & 0 & 18845449 & 0 & -18845449 & -47685439 & on \\
\hline$\rho_{\boldsymbol{n}_{1}, \boldsymbol{x}_{2}}^{\gamma_{4}, 23^{2}}$ & 0 & 0 & 0 & 0 & -34490824 & 0 & 0 & 0 & 0 & 34490824 & $K_{2}$ \\
\hline$\rho_{\boldsymbol{n}_{1}, \boldsymbol{x}_{2}}^{\gamma_{4}, 31}$ & 0 & -17391620 & 12253260 & 0 & 69915146 & -117899406 & -10971678 & -72181722 & 26719220 & 130795200 & \\
\hline $\begin{array}{l}\rho_{\boldsymbol{n}_{1}, \boldsymbol{x}_{2}}^{\gamma_{4}, \omega_{2}} \\
\end{array}$ & 0 & 117647120 & 0 & 0 & 46800340 & 29282384 & 0 & 0 & 0 & 12309516 & \\
\hline$\rho_{\boldsymbol{n}_{1}, \boldsymbol{x}_{2}}^{\gamma_{4}, 11}$ & 12253260 & 0 & 190596948 & 0 & -34173451 & -171438523 & 63948319 & -23837601 & -150987832 & 0 & \\
\hline$\rho_{\boldsymbol{n}_{1}, \boldsymbol{x}_{2}}^{\gamma_{4}, \boldsymbol{x}_{2}}$ & 0 & 0 & 0 & 0 & 82176263 & 0 & 111016253 & 0 & -111016253 & -82176263 & \\
\hline$\rho_{\boldsymbol{n}_{1}, \boldsymbol{x}_{2}}^{\gamma_{4}, 33}$ & 0 & 0 & 0 & 0 & 0 & 0 & 0 & 0 & 0 & 0 & on \\
\hline$\rho_{\boldsymbol{n}_{1}, \boldsymbol{x}_{2}}^{\gamma_{4}, 23^{2}}$ & 0 & 0 & 0 & 0 & 0 & 0 & 92170804 & 0 & -92170804 & 0 & $K_{3}$ \\
\hline$\rho_{\boldsymbol{n}_{1}, \boldsymbol{x}_{2}}^{\gamma_{4}, 31}$ & 0 & 0 & -20253916 & 0 & 0 & -158394664 & 53858088 & 0 & 146028892 & 0 & \\
\hline$\rho_{\boldsymbol{n}_{1}, \boldsymbol{x}_{2}}^{\gamma_{4}, 12}$ & 0 & -36759780 & -348686720 & 0 & -72509332 & 476765190 & -145499112 & 72181722 & 168704198 & 91843194 & \\
\hline$\rho_{\boldsymbol{n}_{1}, \boldsymbol{x}_{2}}^{\gamma_{4}, 11}$ & 12253260 & 36759780 & -12253260 & 263951112 & 65255891 & 24506520 & 47369001 & -203429208 & 3444611 & -351496587 & \\
\hline$\rho_{\boldsymbol{n}_{1}, \boldsymbol{x}_{2}}^{\gamma_{4}, 22}$ & 0 & 110279340 & 0 & 97196160 & -2080783 & -2772867 & 2447827 & 15802163 & 0 & -220871840 & \\
\hline$\rho_{\boldsymbol{n}_{1}, \boldsymbol{x}_{2}}^{\gamma_{4}, 33}$ & 0 & 0 & -36759780 & -19770612 & 0 & 13043859 & 74992528 & 1187909 & -10090231 & -22603673 & on \\
\hline$\rho_{\boldsymbol{n}_{1}, \boldsymbol{x}_{2}}^{\gamma_{4}, 23}$ & 0 & -36759780 & 12253260 & 123717500 & 91843194 & 38742048 & -52777302 & -139383272 & -10971678 & -26663970 & $K_{4}$ \\
\hline$\rho_{\boldsymbol{n}_{1}, \boldsymbol{x}_{2}}^{\gamma_{4}, 31}$ & 0 & 36759780 & 61266300 & 81819360 & -91843194 & -64829766 & -174352686 & 208670186 & 31152140 & -67403720 & \\
\hline$\rho_{\boldsymbol{n}_{1}, \boldsymbol{x}_{2}}^{\gamma_{4}, 12}$ & 0 & -183798900 & -12253260 & -519047460 & -87681628 & -33196314 & 77814112 & 92645702 & 10971678 & 860585430 & \\
\hline
\end{tabular}

The coefficients of $\lambda_{1}^{2}, \lambda_{2}^{2}, \lambda_{3}^{2}, \lambda_{4}^{2}, \lambda_{1} \lambda_{2}, \lambda_{2} \lambda_{3}, \lambda_{3} \lambda_{4}, \lambda_{4} \lambda_{1}, \lambda_{1} \lambda_{3}$ and $\lambda_{2} \lambda_{4}$ for the six piecewise quadratic polynomials $\rho_{\boldsymbol{n}_{1}, \boldsymbol{x}_{2}}^{\gamma_{4}, 11}, \rho_{\boldsymbol{n}_{1}, \boldsymbol{x}_{2}}^{\gamma_{4}, 22}, \rho_{\boldsymbol{n}_{1}, \boldsymbol{x}_{2}}^{\gamma_{4}, 33}, \rho_{\boldsymbol{n}_{1}, \boldsymbol{x}_{2}}^{\gamma_{4}, 23}, \rho_{\boldsymbol{n}_{1}, \boldsymbol{x}_{2}}^{\gamma_{4}, 31}$ and $\rho_{\boldsymbol{n}_{1}, \boldsymbol{x}_{2}}^{\gamma_{4}, 12}$ in (3.38) on each of the four sub-tetrahedra $K_{1}, K_{2}, K_{3}$ and $K_{4}$. We define $\rho_{\boldsymbol{n}_{1}, \boldsymbol{x}_{2}}^{\gamma_{4}, a b}=\rho_{\boldsymbol{n}_{1}, \boldsymbol{x}_{2}}^{\gamma_{4}, b a}$ 


\begin{tabular}{|c|c|}
\hline$\zeta$ & $\Upsilon$ \\
\hline $\boldsymbol{M}_{j j, j j}$ & $\overrightarrow{S_{2,3,4}^{\gamma_{1}, 22}\left(\vec{S}_{2,3,4}^{\gamma_{1}, 22}\right)}$ \\
\hline $\boldsymbol{M}_{j j, k k}$ & $\vec{S}_{2,3,4}^{\gamma_{1}, 22}\left(\vec{S}_{2,3,4}^{\gamma_{1}, 33}\right)^{T}+\vec{S}_{2,3,4}^{\gamma_{1}, 33}\left(\vec{S}_{2,3,4}^{\gamma_{1}, 22}\right)^{T}$ \\
\hline $\boldsymbol{M}_{j j, l l}$ & $\vec{S}_{2,3,4}^{\gamma_{1}, 22}\left(\vec{S}_{2,3,4}^{\gamma_{1}, 44}\right)^{T}+\vec{S}_{2,3,4}^{\gamma_{1}, 44}\left(\vec{S}_{2,3,4}^{\gamma_{1}, 22}\right)^{T}$ \\
\hline $\boldsymbol{M}_{j j, k l}$ & $\vec{S}_{2,3,4}^{\gamma_{1}, 22}\left(\vec{S}_{2,3,4}^{\gamma_{1}, 34}\right)^{T}+\vec{S}_{2,3,4}^{\gamma_{1}, 34}\left(\vec{S}_{2,3,4}^{\gamma_{1}, 22}\right)^{T}$ \\
\hline $\boldsymbol{M}_{j j, l j}$ & $\vec{S}_{2,3,4}^{\gamma_{1}, 22}\left(\vec{S}_{2,3,4}^{\gamma_{1}, 42}\right)^{T}+\vec{S}_{2,3,4}^{\gamma_{1}, 42}\left(\vec{S}_{2,3,4}^{\gamma_{1}, 22}\right)^{T}$ \\
\hline $\boldsymbol{M}_{j j, j k}$ & $\vec{S}_{2,3,4}^{\gamma_{1}, 22}\left(\vec{S}_{2,3,4}^{\gamma_{1}, 23}\right)^{T}+\vec{S}_{2,3,4}^{\gamma_{1}, 23}\left(\vec{S}_{2,3,4}^{\gamma_{1}, 22}\right)^{T}$ \\
\hline $\boldsymbol{M}_{k k, k k}$ & $\vec{S}_{2,3,4}^{\gamma_{1}, 33}\left(\vec{S}_{2,3,4}^{\gamma_{1}, 33}\right)^{T}$ \\
\hline $\boldsymbol{M}_{k k, l l}$ & $\vec{S}_{2,3,4}^{\gamma_{1}, 33}\left(\vec{S}_{2,3,4}^{\gamma_{1}, 44}\right)^{T}+\vec{S}_{2,3,4}^{\gamma_{1}, 44}\left(\vec{S}_{2,3,4}^{\gamma_{1}, 33}\right)^{T}$ \\
\hline $\boldsymbol{M}_{k k, k l}$ & $\vec{S}_{2,3,4}^{\gamma_{1}, 33}\left(\vec{S}_{2,3,4}^{\gamma_{1}, 34}\right)^{T}+\vec{S}_{2,3,4}^{\gamma_{1}, 34}\left(\vec{S}_{2,3,4}^{\gamma_{1}, 33}\right)^{T}$ \\
\hline $\boldsymbol{M}_{k k, l j}$ & $\vec{S}_{2,3,4}^{\gamma_{1}, 33}\left(\vec{S}_{2,3,4}^{\gamma_{1}, 42}\right)^{T}+\vec{S}_{2,3,4}^{\gamma_{1}, 42}\left(\vec{S}_{2,3,4}^{\gamma_{1}, 33}\right)^{T}$ \\
\hline $\boldsymbol{M}_{k k, j k}$ & $\vec{S}_{2,3,4}^{\gamma_{1}, 33}\left(\vec{S}_{2,3,4}^{\gamma_{1}, 23}\right)^{T}+\vec{S}_{2,3,4}^{\gamma_{1}, 23}\left(\vec{S}_{2,3,4}^{\gamma_{1}, 33}\right)^{T}$ \\
\hline$M_{l l, l l}$ & $\vec{S}_{2,3,4}^{\gamma_{1}, 44}\left(\vec{S}_{2,3,4}^{\gamma_{1}, 44}\right)^{T}$ \\
\hline$M_{l l, k l}$ & $\vec{S}_{2,3,4}^{\gamma_{1}, 44}\left(\vec{S}_{2,3,4}^{\gamma_{1}, 34}\right)^{T}+\vec{S}_{2,3,4}^{\gamma_{1}, 34}\left(\vec{S}_{2,3,4}^{\gamma_{1}, 44}\right)^{T}$ \\
\hline $\boldsymbol{M}_{l l, l j}$ & $\vec{S}_{2,3,4}^{\gamma_{1}, 44}\left(\vec{S}_{2,3,4}^{\gamma_{1}, 42}\right)^{T}+\vec{S}_{2,3,4}^{\gamma_{1}, 42}\left(\vec{S}_{2,3,4}^{\gamma_{1}, 44}\right)^{T}$ \\
\hline$M_{l l, j k}$ & $\vec{S}_{2,3,4}^{\gamma_{1}, 44}\left(\vec{S}_{2,3,4}^{\gamma_{1}, 23}\right)^{T}+\vec{S}_{2,3,4}^{\gamma_{1}, 23}\left(\vec{S}_{2,3,4}^{\gamma_{1}, 44}\right)^{T}$ \\
\hline$M_{k l, k l}$ & $\vec{S}_{2,3,4}^{\gamma_{1}, 34}\left(\vec{S}_{2,3,4}^{\gamma_{1}, 34}\right)^{T}$ \\
\hline $\boldsymbol{M}_{k l, l j}$ & $\vec{S}_{2,3,4}^{\gamma_{1}, 34}\left(\vec{S}_{2,3,4}^{\gamma_{1}, 42}\right)^{T}+\vec{S}_{2,3,4}^{\gamma_{1}, 42}\left(\vec{S}_{2,3,4}^{\gamma_{1}, 34}\right)^{T}$ \\
\hline $\boldsymbol{M}_{k l, j k}$ & $\vec{S}_{2,3,4}^{\gamma_{1}, 34}\left(\vec{S}_{2,3,4}^{\gamma_{1}, 23}\right)^{T}+\vec{S}_{2,3,4}^{\gamma_{1}, 23}\left(\vec{S}_{2,3,4}^{\gamma_{1}, 34}\right)^{T}$ \\
\hline$M_{l j, l j}$ & $\vec{S}_{2,3,4}^{\gamma_{1}, 42}\left(\vec{S}_{2,3,4}^{\gamma_{1}, 42}\right)^{T}$ \\
\hline$M_{l j, j k}$ & $\vec{S}_{2,3,4}^{\gamma_{1}, 42}\left(\vec{S}_{2,3,4}^{\gamma_{1}, 23}\right)^{T}+\vec{S}_{2,3,4}^{\gamma_{1}, 23}\left(\vec{S}_{2,3,4}^{\gamma_{1}, 42}\right)^{T}$ \\
\hline $\boldsymbol{M}_{j k, j k}$ & $\vec{S}_{2,3,4}^{\gamma_{1}, 23}\left(\vec{S}_{2,3,4}^{\gamma_{1}, 23}\right)^{T}$ \\
\hline
\end{tabular}

The matrix $\boldsymbol{\zeta}=\frac{1}{|K|} \int_{K} \boldsymbol{\Upsilon} d \boldsymbol{x}$ where the entries in $\mathbf{\Upsilon}$ are in terms of the vectors of piecewise quadratic polynomials defined by (6.1). We note that the matrix $\boldsymbol{\zeta}$ will be identical for every tetrahedron $K$.

[8] I. Babuška, T. Strouboulis, S. K. Gangaraj, K. Copps, and D. K. Datta. Practical aspects of a posteriori estimation for reliable finite element analysis. Comput. 83 Structures, 66(5):627-664, 1998.

[9] M. Bebendorf. A note on the Poincaré inequality for convex domains. Z. Anal. Anwendungen, 22(4):751-756, 2003.

[10] U. Brink and E. Stein. A posteriori error estimation in large-strain elasticity using equilibrated local Neumann problems. Comput. Methods Appl. Mech. Engrg., 161(1-2):77-101, 1998.

[11] C. Carstensen. A unifying theory of a posteriori finite element error control. Numer. Math., 100(4):617-637, 2005.

[12] C. Carstensen and S. A. Funken. Averaging technique for FE-a posteriori error control in elasticity. I. Conforming FEM. Comput. Methods Appl. Mech. Engrg., 190(18-19):2483$2498,2001$.

[13] E. Florentin, L. Gallimard, P. Ladevèze, and J. P. Pelle. Local error estimator for stresses 


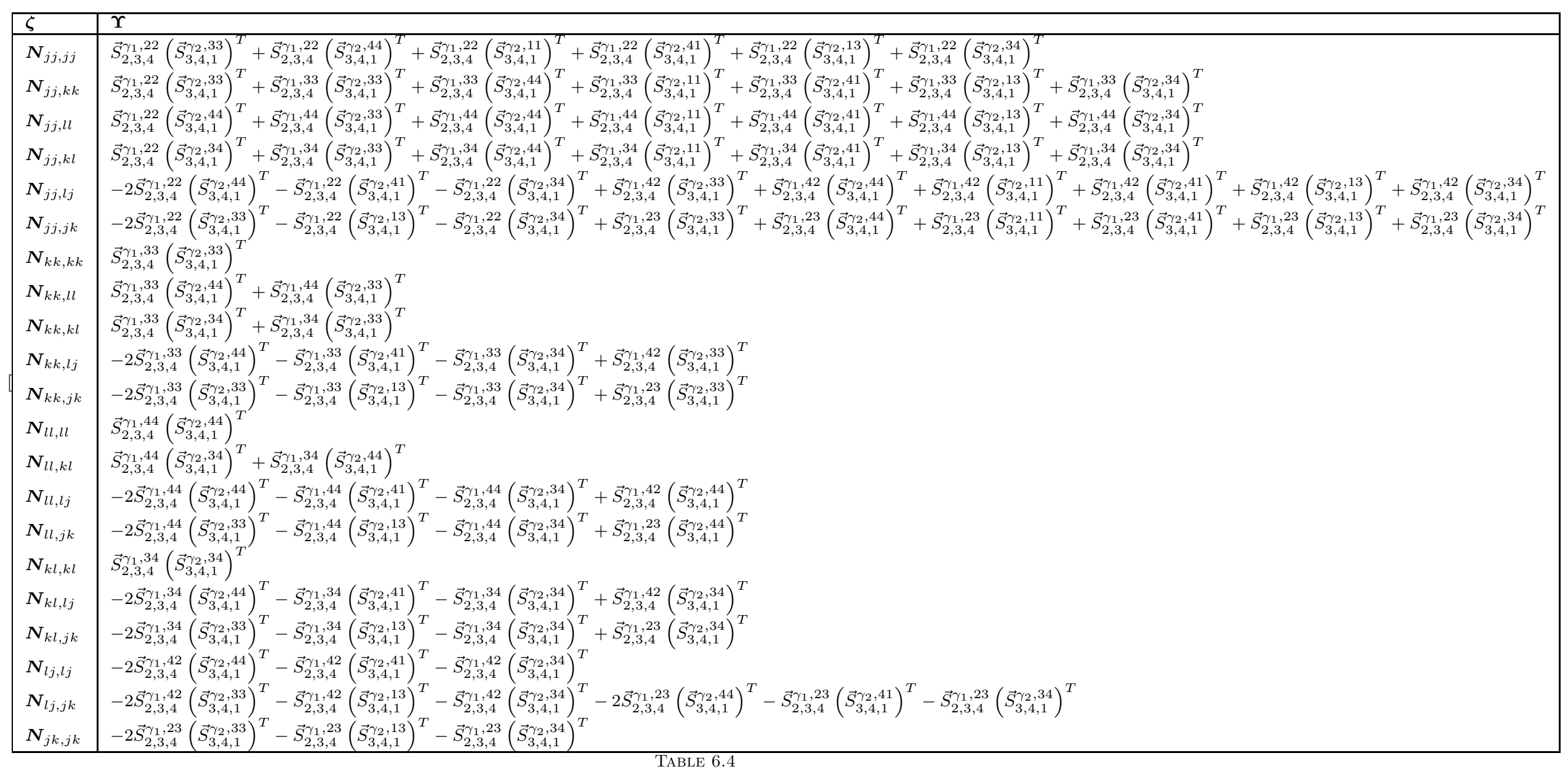

The matrix $\boldsymbol{\zeta}=\frac{1}{|K|} \int_{K} \boldsymbol{\Upsilon} d \boldsymbol{x}$ where the entries in $\boldsymbol{\Upsilon}$ are in terms of the vectors of piecewise quadratic polynomials defined by (6.1). We note that the matrix $\boldsymbol{\zeta}$ will be 
in 3D structural analysis. Comput. \& Structures, 81(18-19):1751-1757, 2003.

[14] C. O. Horgan and L. E. Payne. On inequalities of Korn, Friedrichs and Babuška-Aziz. Arch. Rational Mech. Anal., 82(2):165-179, 1983.

[15] H. Ito. Best constants in Korn-Poincaré's inequalities on a slab. Math. Methods Appl. Sci., 17(7):525-549, 1994.

[16] D. W. Kelly and J. Donovan. Upper bound a posteriori error estimates for the finite element method applied to linear elasticity. In Computational techniques and applications: CTAC-83 (Sydney, 1983), pages 190-206. North-Holland, Amsterdam, 1984.

[17] M. Kempeneers, J.-F. Debongnie, and P. Beckers. Pure equilibrium tetrahedral finite elements for global error estimation by dual analysis. Internat. J. Numer. Methods Engrg., 81(4):513-536, 2010.

[18] S. Nicaise, K. Witowski, and B. I. Wohlmuth. An a posteriori error estimator for the Lamé equation based on equilibrated fluxes. IMA J. Numer. Anal., 28(2):331-353, 2008.

[19] H. Ohtsubo and M. Kitamura. Element by element a posteriori error estimation and improvement of stress solutions for two-dimensional elastic problems. Internat. J. Numer. Methods Engrg., 29(2):223-244, 1990.

[20] H. Ohtsubo and M. Kitamura. Element by element a posteriori error estimation of the finite element analysis for three-dimensional elastic problems. Internat. J. Numer. Methods Engrg., 33(8):1755-1769, 1992.

[21] L. E. Payne and H. F. Weinberger. An optimal Poincaré inequality for convex domains. Arch. Rational Mech. Anal., 5:286-292, 1960.

[22] E. I. Ryzhak. Korn's constant for a parallelepiped with a free face or pair of faces. Math. Mech. Solids, 4(1):35-55, 1999.

[23] R. Verfürth. A Review of A Posteriori Error Estimation and Adaptive Mesh-Refinement Techniques. Wiley-Teubner, 1996.

[24] R. Verfürth. A review of a posteriori error estimation techniques for elasticity problems. Comput. Methods Appl. Mech. Engrg., 176(1-4):419-440, 1999. New advances in computational methods (Cachan, 1997). 